\title{
Efficient Quantum Tomography II
}

\author{
Ryan O’Donnell* \\ Department of Computer Science, Carnegie Mellon \\ University, USA \\ odonnell@cs.cmu.edu
}

\author{
John Wright ${ }^{\dagger}$ \\ Department of Physics, Massachusetts Institute of \\ Technology, USA \\ jswright@mit.edu
}

\begin{abstract}
We continue our analysis of: (i) "Quantum tomography", i.e., learning a quantum state, i.e., the quantum generalization of learning a discrete probability distribution; (ii) The distribution of Young diagrams output by the RSK algorithm on random words. Regarding (ii), we introduce two powerful new tools: first, a precise upper bound on the expected length of the longest union of $k$ disjoint increasing subsequences in a random length- $n$ word with letter distribution $\alpha_{1} \geq \alpha_{2} \geq \cdots \geq \alpha_{d}$. Our bound has the correct main term and second-order term, and holds for all $n$, not just in the large- $n$ limit. Second, a new majorization property of the RSK algorithm that allows one to analyze the Young diagram formed by the lower rows $\lambda_{k}, \lambda_{k+1}, \ldots$ of its output. These tools allow us to prove several new theorems concerning the distribution of random Young diagrams in the nonasymptotic regime, giving concrete error bounds that are optimal, or nearly so, in all parameters. As one example, we give a fundamentally new proof of the celebrated fact that the expected length of the longest increasing sequence in a random length- $n$ permutation is bounded by $2 \sqrt{n}$. This is the $k=1$, $\alpha_{i} \equiv \frac{1}{d}, d \rightarrow \infty$ special case of a much more general result we prove: the expected length of the $k$ th Young diagram row produced by an $\alpha$-random word is $\alpha_{k} n \pm 2 \sqrt{\alpha_{k} d n}$.

From our new analyses of random Young diagrams we derive several new results in quantum tomography, including: (i) learning the eigenvalues of an unknown state to $\epsilon$-accuracy in Hellingersquared, chi-squared, or KL distance, using $n=O\left(d^{2} / \epsilon\right)$ copies; (ii) learning the top- $k$ eigenvalues of an unknown state to $\epsilon$-accuracy in Hellinger-squared or chi-squared distance using $n=O(k d / \epsilon)$ copies or in $\ell_{2}^{2}$ distance using $n=O(k / \epsilon)$ copies; (iii) learning the optimal rank- $k$ approximation of an unknown state to $\epsilon$-fidelity (Hellinger-squared distance) using $n=\widetilde{O}(k d / \epsilon)$ copies. We believe our new techniques will lead to further advances in quantum learning; indeed, they have already subsequently been used for efficient von Neumann entropy estimation.
\end{abstract}

\footnotetext{
*Supported by NSF grant CCF-1618679.

${ }^{\dagger}$ Most of this work performed while affiliated with the Department of Computer Science, Carnegie Mellon University. Partially supported by a Simons Fellowship in Theoretical Computer Science.
}

Permission to make digital or hard copies of all or part of this work for personal or classroom use is granted without fee provided that copies are not made or distributed for profit or commercial advantage and that copies bear this notice and the full citation on the first page. Copyrights for components of this work owned by others than ACM must be honored. Abstracting with credit is permitted. To copy otherwise, or republish, to post on servers or to redistribute to lists, requires prior specific permission and/or a fee. Request permissions from permissions@acm.org.

STOC'17, Montreal, Canada

(C) 2017 ACM. 978-1-4503-4528-6/17/06 ..\$15.00

DOI: $10.1145 / 3055399.3055454$

\section{CCS CONCEPTS}

- Theory of computation $\rightarrow$ Quantum complexity theory; Design and analysis of algorithms;

\section{KEYWORDS}

Quantum tomography, quantum spectrum estimation, longest increasing subsequences, Robinson-Schensted-Knuth algorithm, SchurWeyl duality

\section{ACM Reference format:}

Ryan O'Donnell and John Wright. 2017. Efficient Quantum Tomography II. In Proceedings of 49th Annual ACM SIGACT Symposium on the Theory of Computing, Montreal, Canada, fune 2017 (STOC'17), 13 pages.

DOI: $10.1145 / 3055399.3055454$

\section{INTRODUCTION}

The Robinson-Schensted-Knuth (RSK) algorithm is a well-known combinatorial algorithm with diverse applications throughout mathematics, computer science, and physics. Given a word $w$ with $n$ letters from the alphabet $[d]$, it outputs two semistandard Young tableaus $(P, Q)=\operatorname{RSK}(w)$ with common shape given by some Young diagram $\lambda \in \mathbb{N}^{d}\left(\lambda_{1} \geq \cdots \geq \lambda_{d}\right)$. We write $\lambda=\operatorname{shRSK}(w)$, and mention that $\lambda$ can be defined independently of the RSK algorithm as in Theorem 1.2 below. In the RSK algorithm, the process generating the first row is sometimes called patience sorting, and it is equivalent to the basic dynamic program for computing $w$ 's longest (weakly) increasing subsequence.

Definition 1.1. Given a word $w \in[d]^{n}$, a subsequence is a sequence of letters $\left(w_{i_{1}}, \ldots, w_{i_{\ell}}\right)$ such that $i_{1}<\cdots<i_{\ell}$. The length of the subsequence is $\ell$. We say that the subsequence is weakly increasing, or just increasing, if $w_{i_{1}} \leq \cdots \leq w_{i_{\ell}}$. We write LIS $(w)$ for the length of the longest weakly increasing subsequence in $w$.

Hence $\lambda_{1}=\operatorname{LIS}(w)$, a result known as Schensted's Theorem [Sch61]. Further rows of $\lambda$ are characterized by Greene's Theorem as giving the "higher order LIS statistics" of $w$.

THeOREM 1.2 ([GRE74]). Suppose $\lambda=\operatorname{shRSK}(w)$. Then for each $k$, $\lambda_{1}+\cdots+\lambda_{k}$ is equal to the length of the longest union of $k$ disjoint (weakly) increasing subsequences in $w$.

For background on the RSK algorithm, see e.g. [Ful97, Rom14] and the references therein.

Many applications involve studying the behavior of the RSK algorithm when its input is drawn from some random distribution. A famous case is the uniform distribution over length- $n$ permutations $\pi \sim S_{n}$ (in which case $d=n$ ); here the resulting random Young diagram $\lambda=\operatorname{RSK}(\pi)$ is said to have Plancherel distribution. Starting 
with the work of Ulam [Ula61], a line of research has studied the distribution of the longest increasing subsequence of $\boldsymbol{\pi}$; its results are summarized as follows: E[LIS $(\pi)] \rightarrow 2 \sqrt{n}$ as $n \rightarrow \infty$ [LS77, VK77] (in fact, $\mathrm{E}[\operatorname{LIS}(\boldsymbol{\pi})] \leq 2 \sqrt{n}$ for all $n$ [VK85, Pil90]), and the deviations of $\operatorname{LIS}(\pi)$ from this value can be characterized by the Tracy-Widom distribution from random matrix theory [BDJ99]. The RSK algorithm has played a central role in many of these developments, and these results have been shown to apply not just to the first row $\lambda_{1}=\operatorname{LIS}(\pi)$ but also to the entire shape of $\lambda$ [Joh01, BOO00]. In a different stream of research, the Plancherel distribution arises naturally in quantum algorithms which perform Fourier sampling over the symmetric group. Here, its properties have been used to show that any quantum algorithm for graph isomorphism (or, more generally, the hidden subgroup problem on the symmetric group) which uses the "standard approach" must perform highly entangled measurements across many copies of the coset state [HRTS03, MRS08, $\mathrm{HMR}^{+} 10$ ].

In this work, we consider a more general setting, sometimes called the inhomogeneous random word model, in which the input to the RSK algorithm is a random word $w$ whose letters are selected independently from some probability distribution.

Definition 1.3. Given a probability distribution $\alpha=\left(\alpha_{1}, \ldots, \alpha_{d}\right)$ on alphabet $[d]$, an $n$-letter $\alpha$-random word $\boldsymbol{w}=\left(\boldsymbol{w}_{1}, \ldots, \boldsymbol{w}_{n}\right)$, written as $\boldsymbol{w} \sim \alpha^{\otimes n}$, is a random word in which each letter $\boldsymbol{w}_{i}$ is independently drawn from $[d]$ according to $\alpha$. The Schur-Weyl distribution $\mathrm{SW}^{n}(\alpha)$ is the distribution on Young diagrams given by $\lambda=\operatorname{shRSK}(w)$. Although it is not obvious, it is a fact that the distribution $\mathrm{SW}^{n}(\alpha)$ does not depend on the ordering of $\alpha$ 's components. Thus unless otherwise stated, we will assume that $\alpha$ is sorted; i.e., $\alpha_{1} \geq \cdots \geq \alpha_{d}$.

(The homogeneous random word model is the special case in which $\alpha_{i}=\frac{1}{d}$, for each $i \in[d]$. It is easy to see that in this case, $\operatorname{SW}^{n}(\alpha)$ converges to the Plancherel distribution as $d \rightarrow \infty$.) Aside from arising naturally in combinatorics and representation theory, the Schur-Weyl distribution also appears in a large number of problems in quantum learning and data processing, as we will see below.

Much of the prior work on the Schur-Weyl distribution has occurred in the asymptotic regime, in which $d$ and $\alpha$ are held constant and $n \rightarrow \infty$. An easy exercise in Chernoff bounds shows that $\operatorname{LIS}(\boldsymbol{w}) / n \rightarrow \alpha_{1}$ as $n \rightarrow \infty$. Generalizing this, a sequence of works [TW01, Joh01, ITW01, HX13, Mél12] have shown that in this regime, $\lambda$ is equal to $\left(\alpha_{1} n, \ldots, \alpha_{d} n\right)$ plus some lower-order fluctuations distributed as the eigenvalues of certain random matrix ensembles. From these works, we may extract the following ansatz, coarsely describing the limiting behavior of the rows of $\lambda$.

$$
\text { Ansatz: For all } k \in[d], \lambda_{k} \approx \alpha_{k} n \pm 2 \sqrt{\alpha_{k} d_{k} n} \text {. }
$$

Here $d_{k}$ is the number of times $\alpha_{k}$ occurs in $\left(\alpha_{1}, \ldots, \alpha_{d}\right)$. We survey this literature below in Section 1.5.

\subsection{A Nonasymptotic Theory of the Schur-Weyl Distribution}

In this work, motivated by problems in quantum state learning, we study the Schur-Weyl distribution in the nonasymptotic regime. Previous efforts in this direction were the works [HM02, CM06] and, more extensively, our previous paper [OW16]. Our goal is to prove worst-case bounds on the shape of $\lambda$ which hold for all $n$, independent of $d$ and $\alpha$. When possible, we would like to translate certain features of the Schur-Weyl distribution present in the asymptotic regime - in particular, the ansatz and its consequences down into the nonasymptotic regime.

Clearly, nonasymptotic results cannot depend on the quantity $d_{k}$, which can be sensitive to arbitrarily small changes in $\alpha$ that are undetectable when $n$ is small. (Consider especially when $\alpha$ is uniform versus when $\alpha$ is uniform but with each entry slightly perturbed.) Instead, our results are in terms of the quantity $\min \left\{1, \alpha_{k} d\right\}$, for each $k \in[d]$, which always upper bounds $\alpha_{k} d_{k}$.

Our first result tightly bounds the expected row lengths, in line with the ansatz.

Theorem 1.4. For $k \in[d]$, set $v_{k}=\min \left\{1, \alpha_{k} d\right\}$. Then

$$
\alpha_{k} n-2 \sqrt{v_{k} n} \leq \underset{\lambda \sim \mathrm{SW}^{n}(\alpha)}{\mathrm{E}} \lambda_{k} \leq \alpha_{k} n+2 \sqrt{v_{k} n},
$$

This improves on a result from [OW16], which showed an upper bound in the $k=1$ case with error $+2 \sqrt{2} \sqrt{n}$ for general $\alpha$ and with error $+2 \sqrt{n}$ for $\alpha$ the uniform distribution. Setting $\alpha=\left(\frac{1}{d}, \ldots \frac{1}{d}\right)$ and letting $d \rightarrow \infty$, the $k=1$ case of Theorem 1.4 recovers the above-mentioned celebrated fact that the length of the longest increasing subsequence of a random permutation of $n$ is at most $2 \sqrt{n}$ in expectation. Our result gives only the second proof of this statement since it was originally proved independently by Vershik and Kerov in 1985 [VK85] and by Pilpel in 1990 [Pil90]. Next, we bound the mean-squared error of the estimator $\lambda_{k} / n$ for $\alpha_{k}$.

Theorem 1.5. For $k \in[d]$, set $v_{k}=\min \left\{1, \alpha_{k} d\right\}$. Then

$$
\underset{\lambda \sim \mathrm{SW}^{n}(\alpha)}{\mathrm{E}}\left(\lambda_{k}-\alpha_{k} n\right)^{2} \leq O\left(v_{k} n\right) .
$$

Again, this is in line with the ansatz. This theorem can be used to derive tight bounds (up to constant factors) on the convergence of the normalized Young diagram $\underline{\lambda}=\left(\lambda_{1} / n, \ldots, \lambda_{d} / n\right)$ to $\alpha$ in a variety of distance measures, including Hellinger-squared distance and the KL and chi-squared divergences. Now in fact, using related techniques, in [OW16] we were able to prove convergence bounds for some distance measures with stronger constants:

TheOREM 1.6 ([OW16]).

$$
\underset{\lambda \sim \mathrm{SW}^{n}(\alpha)}{\mathbf{E}}\|\underline{\boldsymbol{\lambda}}-\alpha\|_{2}^{2} \leq \frac{d}{n} \text { and } \underset{\lambda \sim \mathrm{SW}^{n}(\alpha)}{\mathbf{E}}\|\underline{\boldsymbol{\lambda}}-\alpha\|_{1} \leq \frac{d}{\sqrt{n}} .
$$

In this work, we extend Theorem 1.6 to other, more challenging distance measures.

Theorem 1.7. Let $d(\cdot, \cdot)$ be any of $d_{\mathrm{H}^{2}}(\cdot, \cdot), d_{\mathrm{KL}}(\cdot, \cdot)$, or $d_{\chi^{2}}(\cdot, \cdot)$. Then $\underset{\lambda \sim S W^{n}(\alpha)}{\mathbf{E}} d(\underline{\lambda}, \alpha) \leq \frac{d^{2}}{n}$.

Not only are Theorems 1.6 and 1.7 in line with the ansatz, they even have the correct constant factors, as predicted below by Theorem 1.24 in the asymptotic regime.

Finally, we show similar results for truncated distances, in which only the top $k$ entries of $\lambda$ and the top $k$ entries of $\alpha$ are compared with each other. In [OW16], this was carried out for truncated $\ell_{1}$ distance.

$$
\text { Theorem } 1.8 \text { ([OW16]). } \underset{\lambda \sim \mathrm{SW}^{n}(\alpha)}{\mathrm{E}} d_{\mathrm{TV}}^{(k)}(\underline{\boldsymbol{\lambda}}, \alpha) \leq \frac{1.92 k+.5}{\sqrt{n}} .
$$


By following the proof of this result, our Theorem 1.4 immediately implies the same bound with 1.5 in place of 1.92. In addition, we prove similar bounds for truncated $\ell_{2}^{2}$, Hellinger, and chi-squared distances.

THeOREM 1.9. $\underset{\lambda \sim \mathrm{SW}^{n}(\alpha)}{\mathrm{E}} d_{\ell_{2}^{2}}^{(k)}(\underline{\lambda}, \alpha) \leq \frac{46 k}{n}$.

Theorem 1.10. Let $d(\cdot, \cdot)$ be either $d_{\mathrm{H}^{2}}^{(k)}(\cdot, \cdot)$ or $d_{\chi^{2}}^{(k)}(\cdot, \cdot)$. Then

$$
\underset{\lambda \sim \mathrm{SW}^{n}(\alpha)}{\mathrm{E}} d(\underline{\lambda}, \alpha) \leq \frac{46 k d}{n} .
$$

These results follow the ansatz, though our techniques are not yet strong enough to achieve optimal constant factors.

\subsection{Techniques}

Our main techniques include a pair of majorization theorems for the RSK algorithm. Here we refer to the following definition.

Definition 1.11. For $x, y \in \mathbb{R}^{d}$, we say that $x$ majorizes $y$, denotes $x>y$ if $x_{[1]}+\cdots+x_{[k]} \geq y_{[1]}+\cdots+y_{[k]}$ for all $k \in[d]$, with equality for $k=d$. Here the notation $x_{[i]}$ means the $i$ th largest value among the $x_{j}$ 's. In the case of Young diagrams $\lambda$ and $\mu$, we also use the standard notation $\lambda \unrhd \mu$. Weak majorization, denoted with either $>_{w}$ or $\unrhd_{w}$, is the case when the equality constraint may not necessarily hold.

Several of our results require understanding the behavior of an individual row $\lambda_{k}$, for $k \in[d]$. However, the RSK algorithm's sequential behavior makes understanding rows after the first quite difficult. So instead, we adopt the strategy of proving bounds only for the first row (which can sometimes be done directly), and then translating them to the $k$ th row via the following new theorem.

Theorem 1.12. Fix an integer $k \geq 1$ and an ordered alphabet $\mathcal{A}$. Consider the RSK algorithm applied to some string $x \in \mathcal{A}^{n}$. During the course of the algorithm, some letters of $x$ get bumped from the $k$ th row and inserted into the $(k+1)$ th row. Let $x^{(k)}$ denote the string formed by those letters in the order they are so bumped. On the other hand, let $\bar{x}$ be the subsequence of $x$ formed by the letters of $x^{(k)}$ in the order they appear in $x$. Then $\operatorname{shRSK}(\bar{x}) \unrhd \operatorname{shRSK}\left(x^{(k)}\right)$.

Our other key tool is the following result allowing us to bound how much larger $\lambda_{1}+\cdots+\lambda_{k}$ is than its intended value $\alpha_{1} n+$ $\cdots+\alpha_{k} n$ in expectation.

THEOREM 1.13. Let $\alpha$ be a sorted probability distribution on $[d]$ and let $k \in[d]$. Then for all $n \in \mathbb{N}$,

$$
\begin{aligned}
\mathrm{E}\left[\sum_{i=1}^{k} \lambda_{i}^{(n)}\right]-\sum_{i=1}^{k} \alpha_{i} n & \leq \operatorname{Excess}_{k}(\alpha) \\
& \text { where } \operatorname{Excess}_{k}(\alpha)=\sum_{i \leq k<j} \frac{\alpha_{j}}{\alpha_{i}-\alpha_{j}} .
\end{aligned}
$$

Furthermore, using the notation $E_{k}^{(n)}(\alpha)$ for the left-hand side, it holds that $E_{k}^{(n)}(\alpha) \nearrow \operatorname{Excess}_{k}(\alpha)$ as $n \rightarrow \infty$ provided that all $\alpha_{i}$ 's are distinct.
The fact that $E_{1}^{(n)}(\alpha) \rightarrow \operatorname{Excess}_{1}(\alpha)$ as $n \rightarrow \infty$ when all the $\alpha_{i}$ 's are fixed and distinct was originally proven by Its, Tracy, and Widom [ITW01]. We extend this to the general $k$ case, and also show that the sequence $E_{1}^{(n)}(\alpha)$ is increasing in $n$, so that $\operatorname{Excess}_{k}(\alpha)$ is an upper bound for all $n$. So long as $\alpha_{k}$ and $\alpha_{k+1}$ are sufficiently separated we have found that $\operatorname{Excess}_{k}(\alpha)$ gives a surprisingly accurate bound on $\mathrm{E}\left[\lambda_{1}+\cdots+\lambda_{k}\right]-\left(\alpha_{1} n+\cdots+\alpha_{k} n\right)$. When $\alpha_{k}$ and $\alpha_{k+1}$ are not well-separated, on the other hand, $\operatorname{Excess}_{k}(\alpha)$ can be arbitrarily large. In this case, we consider a mildly perturbed distribution $\alpha^{\prime}$ in which $\alpha_{k}^{\prime}$ and $\alpha_{k+1}^{\prime}$ are well-separated and then apply Theorem 1.13 to $\alpha^{\prime}$ instead. Supposing that $\alpha^{\prime}>\alpha$, we may then relate the bounds we get on $\operatorname{SW}^{n}\left(\alpha^{\prime}\right)$ back to $\mathrm{SW}^{n}(\alpha)$ using Theorem 1.11 from [OW16].

Theorem 1.14 ([OW16]). Let $\alpha, \beta \in \mathbb{R}^{d}$ be sorted probability distributions with $\beta>\alpha$. Then for any $n \in \mathbb{N}$ there is a coupling $(\boldsymbol{\lambda}, \boldsymbol{\mu})$ of $\mathrm{SW}^{n}(\alpha)$ and $\mathrm{SW}^{n}(\beta)$ such that $\boldsymbol{\mu} \unrhd \boldsymbol{\lambda}$ always.

\subsection{Quantum State Learning}

Our main application of these bounds is to problems in the area of quantum state learning. Here, one is given $n$ copies of a mixed state $\rho \in \mathbb{C}^{d \times d}$ and asked to learn some property of $\rho$. For example, one might attempt to learn the entire $d \times d$ matrix (quantum tomography), just its spectrum $\alpha=\left(\alpha_{1}, \ldots, \alpha_{d}\right)$ (quantum spectrum estimation), or some other more specific property such as its von Neumann entropy, its purity, and so forth. These problems play key roles in various quantum computing applications, including current-day verification of experimental quantum devices and hypothesized future quantum protocols such as entanglement verification. We allow ourselves arbitrary entangled measurements, and our goal is to learn while using as few copies $n$ as possible.

The standard approach to designing entangled measurements for quantum state learning [ARS88, KW01] uses a powerful tool from representation theory called Schur-Weyl duality, which states that

$$
\left(\mathbb{C}^{d}\right)^{\otimes n} \cong \bigoplus_{\lambda} \mathrm{Sp}_{\lambda} \otimes \mathrm{V}_{\lambda}^{d}
$$

Here the direct sum ranges over all partitions $\lambda \vdash n$ of height at most $d$, and $\mathrm{Sp}_{\lambda}$ and $\mathrm{V}_{\lambda}^{d}$ are the irreps of the symmetric and general linear groups corresponding to $\lambda$. Measuring $\rho^{\otimes n}$ according to the projectors $\left\{\Pi_{\lambda}\right\}_{\lambda}$ corresponding to the $\lambda$-subspaces is called weak Schur sampling and is the optimal measurement if one is interested only in learning $\rho$ 's spectrum $\alpha$ (or some function of $\alpha$ ). The outcome of this measurement is a random $\lambda$ whose distribution depends only on $\alpha$; in fact:

FACT 1.15. When performed on $\rho^{\otimes n}$, the measurement outcome $\lambda$ of weak Schur sampling is distributed exactly as the Schur-Weyl distribution $\mathrm{SW}^{n}(\alpha)$, where $\alpha$ is $\rho$ 's spectrum.

(See, for example, the discussion of this in [OW16].) Following weak Schur sampling, $\rho^{\otimes n}$ collapses to the subspace corresponding to $\lambda$, and if one wishes to learn about more than just $\rho$ 's spectrum, one must perform a further measurement within this subspace. An algorithm which does so is said to have performed strong Schur sampling. Note that weak Schur sampling refers to a specific measurement, whereas strong Schur sampling refers to a class of measurements. 
Fact 1.15, when paired with our results from Section 1.1, immediately suggests the following algorithm for estimating $\rho$ 's spectrum: perform weak Schur sampling, receive the outcome $\lambda$, and output $\underline{\lambda}$. This is exactly the empirical Young diagram (EYD) algorithm introduced independently by Alicki, Ruckinci, and Sadowski [ARS88] and Keyl and Werner [KW01]. To date, this is the best known spectrum estimation algorithm, and it has recently been proposed for current-day experimental implementation $\left[\mathrm{BAH}^{+} 16\right]$. Our Theorem 1.7 immediately implies the following.

THeOREм 1.16. The spectrum $\alpha$ can be learned in Hellinger-squared distance, KL divergence, and chi-squared divergence using $n=O\left(d^{2} / \epsilon\right)$ copies.

Previously, it was known from the works of Hayashi and Matsumoto [HM02] and Christandl and Mitcheson [CM06] that $n=$ $O\left(d^{2} / \epsilon\right) \cdot \log \left(d / \epsilon^{2}\right)$ copies sufficed for KL divergence (and hence for Hellinger-squared). We note that Theorem 1.6 from [OW16] gave learning bounds of $O(d / \epsilon)$ and $O\left(d^{2} / \epsilon^{2}\right)$ for spectrum learning under $\ell_{2}^{2}$ and $\ell_{1}$ distance, respectively. Combined with the lower bound from [OW15] showing that the EYD algorithm requires $n=\Omega\left(d^{2} / \epsilon^{2}\right)$ copies for $\ell_{1}$ learning, we have given optimal bounds for the EYD algorithm in terms of all five distance metrics.

For the more difficult problem of quantum tomography, the optimal number of copies needed to learn $\rho$ in trace distance was recently determined to be $n=\Theta\left(d^{2} / \epsilon^{2}\right)$ - the upper bound from our previous work [OW16] and the lower bound from the independent work of Haah et al. $\left[\mathrm{HHJ}^{+} 16\right]$. The optimal complexity of learning $\rho$ in infidelity - i.e., outputting an estimate $\widehat{\boldsymbol{\rho}}$ such that $1-F(\rho, \widehat{\boldsymbol{\rho}}) \leq \epsilon$ - remains open, however. Essentially the best prior result is by Haah et al. $\left[\mathrm{HHJ}^{+} 16\right]$, who showed that $n=O\left(d^{2} / \epsilon\right) \cdot \log (d / \epsilon)$ copies suffice. For our results, we find it convenient to work with the very closely related quantum Hellinger-squared distance $d_{\mathrm{H}^{2}}(\cdot, \cdot)$. This is known to be the same as infidelity $1-F(\rho, \widehat{\rho})$ up to a factor of 2 (see Section 2 for details), and hence learning in quantum Hellinger-squared distance is equivalent to learning in infidelity up to a small constant. We show the following theorem.

Theorem 1.17. A state $\rho \in \mathbb{C}^{d \times d}$ can be learned in quantum Hellinger-squared distance with copy complexity

$$
n=O\left(\min \left\{\frac{d^{2}}{\epsilon} \log \left(\frac{d}{\epsilon}\right), \frac{d^{3}}{\epsilon}\right\}\right) .
$$

The left-hand term in the min gives a new proof of the fidelity bound of Haah et al. $\left[\mathrm{HHJ}^{+} 16\right]$. The right-hand term in the min is new; previously it was known only how to learn $\rho$ in fidelity using $n=O(h(d) / \epsilon)$ copies for some unspecified function $h(\cdot)$ (see the list of citations in $\left.\left[\mathrm{HHJ}^{+} 16\right]\right)$. Along with our trace distance bound of $n=O\left(d^{2} / \epsilon^{2}\right)-$ which implies a fidelity bound of $O\left(d^{2} / \epsilon^{2}\right)-$ we now have three incomparable upper bounds on the complexity of fidelity tomography, none of which match the best known lower bound of $\Omega\left(d^{2} / \epsilon\right)$ from $\left[\mathrm{HHJ}^{+} 16\right]$. Settling the complexity of fidelity learning remains an important open problem.

To perform full-state tomography, we analyze Keyl's algorithm [Key06]. After performing weak Schur sampling and receiving a random $\lambda$, it performs a subsequent measurement in the $\lambda$-subspace whose measurement outcomes correspond to $d \times d$ unitary matrices. We denote by $\mathrm{K}_{\lambda}(\rho)$ the distribution on unitary matrices observed given $\lambda$ and $\rho$. The algorithm receives a random $V \sim \mathrm{K}_{\lambda}(\rho)$ from this measurement and then outputs the density matrix $V \operatorname{diag}(\lambda / n) V^{\dagger}$. We will only require one fact about this algorithm from [OW16], and so we defer the full description of Keyl's measurement and algorithm to the papers [Key06, OW16].

\subsection{Principal Component Analysis}

Next, we consider natural "principal component analysis" (PCA)style versions of the above problems. Here, rather than learning the whole state or spectrum, the goal is to learn the "largest" $k$ dimensional part of the state or spectrum. These problems arise naturally when the state is "fundamentally" low rank, but has been perturbed by a small amount of noise. For spectrum estimation, this involves learning the first $k \alpha_{i}$ 's under the ordering $\alpha_{1} \geq \cdots \geq \alpha_{d}$. Previous work [OW16] used Theorem 1.8 to learn the first $k \alpha_{i}$ 's in trace distance using $n=O\left(k^{2} / \epsilon^{2}\right)$ copies. Using our Theorems 1.9 and 1.10, we extend this result to other distance measures.

THeORem 1.18. The first $k \alpha_{i}$ 's can be learned in Hellinger-squared distance or chi-squared divergence using $n=O(k d / \epsilon)$ copies, and in $\ell_{2}^{2}$ distance using $n=O(k / \epsilon)$ copies.

For full-state PCA, the natural variant is to output a rank- $k$ matrix $\widehat{\rho}$ which is almost as good as the best rank- $k$ approximation to $\rho$. For trace distance, the work of [OW16] showed that $n=$ $O\left(k d / \epsilon^{2}\right)$ copies are sufficient to output an estimate with error at most $\epsilon$ more than the error of the best rank- $k$ approximation. In this work, we show the following fidelity PCA result.

Theorem 1.19. There is an algorithm that, for any $\rho \in \mathbb{C}^{d \times d}$ and $k \in[d]$, outputs a random rank-k (or less) hypothesis $\widehat{\boldsymbol{\rho}}$ such that

$$
\mathbf{E}\left[D_{\mathrm{H}^{2}}(\hat{\boldsymbol{\rho}}, \rho)\right] \leq \alpha_{>k}+O\left(\frac{k d L}{n}\right)+O\left(k L \sqrt{\frac{\alpha_{>k}}{n}}\right),
$$

where $L=\min \{k, \ln n\}$ and $\alpha_{>k}=\alpha_{k+1}+\cdots+\alpha_{d}$.

Let us spend time interpreting this result. The Hellinger-squared error of the best rank- $k$ approximation to $\rho-$ the projection of $\rho$ to its top- $k$ eigenspace - is given by $\alpha_{>k}$. When $\rho$ is exactly of rank $k$, then $\alpha_{>k}=0$, and this bound tells us that

$$
n=O\left(\min \left\{\frac{k d}{\epsilon} \log \left(\frac{d}{\epsilon}\right), \frac{k^{2} d}{\epsilon}\right\}\right)
$$

copies are sufficient to learn $\rho$ up to error $\epsilon$. The left-hand term in the min was shown previously by Haah et al. $\left[\mathrm{HHJ}^{+} 16\right]$ using different techniques, whereas the right-hand term is new. In the case that $\rho$ is not rank- $k$, let us first make the reasonable assumption that $k \leq d / \ln n$. Then

$$
\begin{aligned}
& \mathrm{E}\left[D_{\mathrm{H}^{2}}(\widehat{\boldsymbol{\rho}}, \rho)\right] \leq \alpha_{>k}+Z_{1}+Z_{2}, \\
& \text { where } Z_{1}=O\left(\frac{k d \ln n}{n}\right), Z_{2}=O\left(\sqrt{\frac{\alpha_{>k} k d \ln n}{n}}\right) .
\end{aligned}
$$

Noting that $Z_{2}$ is the geometric mean of $\alpha_{>k}$ and $Z_{2}$, we get that for any $\delta>0$,

$$
\mathbf{E}\left[D_{\mathrm{H}^{2}}(\widehat{\boldsymbol{\rho}}, \rho)\right] \leq(1+\delta) \cdot \alpha_{>k}+O_{\delta}\left(\frac{k d \ln n}{n}\right) .
$$

Hence, this tells us that $n=O(k d / \epsilon) \cdot \log (d / \epsilon)$ copies are sufficient to learn $\rho$ to error $(1+\delta) \cdot \alpha_{>k}+\epsilon$ (essentially recovering the exactly rank- $k$ case). Finally, in the unlikely case of $k>d / \ln n$, a similar 
argument shows that $n=O(k d / \epsilon) \cdot \log ^{2}(d / \epsilon)$ copies are sufficient to learn $\rho$ to error $(1+\delta) \cdot \alpha_{>k}+\epsilon$.

\subsection{Asymptotics of the Schur-Weyl Distribution}

In this section, we survey the known results on the Schur-Weyl distribution in the asymptotic setting. Though we are primarily interested in proving convergence results with explicit error bounds in the nonasymptotic setting, the asymptotic regime is useful for understanding the high-level features of the Schur-Weyl distribution. Indeed, the early quantum computing papers [ARS88, KW01] on this topic operated in this regime.

The earliest theorem in this area is due to Vershik and Kerov [VK81], who showed the following:

THEOREM 1.20 ([VK81]). Let $\alpha=\left(\alpha_{1}, \ldots, \alpha_{d}\right)$ be a sorted probability distribution, and let $\lambda \sim \mathrm{SW}^{n}(\alpha)$. Then for all $k \in[d]$, as $n \rightarrow \infty$ we have $\lambda_{k} / n \rightarrow \alpha_{k}$ in probability.

This theorem has been reproven in a variety of works, including independently by [ARS88] and [KW01] in the quantum computing literature.

Subsequent work determined the lower-order asymptotics of the Schur-Weyl distribution. As it turns out, the qualitative features of the distribution depend on whether $\alpha$ has any repeated values. The simplest case, when all the $\alpha_{i}$ 's are distinct, was first handled in the work of Alicki, Rudnicki, and Sadowski [ARS88].

Theorem 1.21 ([ARS88]). Let $\alpha=\left(\alpha_{1}, \ldots, \alpha_{d}\right)$ be a sorted probability distribution in which every entry is distinct. Let $\lambda \sim \operatorname{SW}^{n}(\alpha)$, and let $\left(\boldsymbol{g}_{1}, \ldots, \boldsymbol{g}_{d}\right)$ be centered jointly Gaussian random variables with $\operatorname{Var}\left[\boldsymbol{g}_{i}\right]=\alpha_{i}\left(1-\alpha_{i}\right)$ and $\operatorname{Cov}\left[\boldsymbol{g}_{i}, \boldsymbol{g}_{j}\right]=-\alpha_{i} \alpha_{j}$, for $i \neq j .{ }^{1}$ Then as $n \rightarrow \infty$,

$$
\left(\frac{\lambda_{1}-\alpha_{1} n}{\sqrt{n}}, \ldots, \frac{\lambda_{d}-\alpha_{d} n}{\sqrt{n}}\right) \rightarrow\left(g_{1}, \ldots, g_{d}\right)
$$

in distribution.

In other words, in the case of distinct $\alpha_{i}$ 's, the Schur-Weyl distribution acts in the asymptotic regime like the multinomial distribution with parameter $\alpha$. The intuition is that given an $\alpha$-random word $\boldsymbol{w}$, the count of 1's is so much greater than the count of any other letter that the longest increasing subsequence is not much longer than the all-1's subsequence. Similarly, the longest pair of disjoint increasing subsequences is not much longer than the all-1's and all-2's subsequences, and so forth. This theorem has been reproven many times, such as by [HX13, Buf12, Mél12, FMN13].

On the other hand, when $\alpha$ is degenerate, i.e. the $\alpha_{i}$ 's are not all distinct, then $\mathrm{SW}^{n}(\alpha)$ has a surprisingly non-Gaussian limiting behavior. The first paper along this line of work was by Baik, Deift, and Johannson [BDJ99]; it characterized the Plancherel distribution (a special case of the Schur-Weyl distribution) in terms of the eigenvalues of the Gaussian unitary ensemble.

Definition 1.22. The Gaussian unitary ensemble $\mathrm{GUE}_{d}$ is the distribution on $d \times d$ Hermitian matrices $X$ in which (i) $X_{i, i} \sim \mathcal{N}(0,1)$ for each $i \in[d]$, and (ii) $X_{i, j} \sim \mathcal{N}(0,1)_{\mathbb{C}}$ and $X_{j, i}=\overline{X_{i, j}}$ for all $i<j \in[d]$. Here $\mathcal{N}(0,1)_{\mathbb{C}}$ refers to the complex standard Gaussian,

\footnotetext{
${ }^{1}$ This is a degenerate Gaussian distribution, supported on $\sum_{i} \boldsymbol{g}_{i}=0$
}

distributed as $\mathcal{N}\left(0, \frac{1}{2}\right)+i \mathcal{N}\left(0, \frac{1}{2}\right)$. The traceless GUE, denoted GUE ${ }_{d}^{0}$, is the probability distribution on $d \times d$ Hermitian matrices $Y$ given by

where $X \sim \mathrm{GUE}_{d}$.

$$
Y=X-\frac{\operatorname{tr}(X)}{d} \cdot I
$$

The next fact characterizes the eigenvalues of the traceless GUE in the limit (cf. [HX13]).

FACT 1.23. Given $Y \sim \operatorname{GUE}_{d}^{0}$, then as $d \rightarrow \infty$,

$$
\left(\frac{\operatorname{eig}_{1}(Y)}{\sqrt{d}}, \ldots, \frac{\operatorname{eig}_{d}(Y)}{\sqrt{d}}\right)
$$

converges almost surely to the semicircle law with density $\sqrt{4-x^{2}} / 2 \pi$, $-2 \leq x \leq 2$, where $\operatorname{eig}_{i}(Y)$ denotes the ith largest eigenvalue of $Y$.

The traceless GUE was first used to characterize the Schur-Weyl distribution in the special case when $\alpha$ is the uniform distribution, the homogeneous random word case. In this case, Tracy and Widom [TW01] showed such a characterization for just the first row $\lambda_{1}$, and Johansson [Joh01] extended their result to hold for the entire diagram $\lambda$, as follows (cf. the quantum mechanical proof of this theorem by Kuperberg [Kup02]).

Theorem 1.24 ([Јон01]). Let $\alpha=\left(\frac{1}{d}, \ldots, \frac{1}{d}\right)$ be the uniform distribution. Let $\lambda \sim \operatorname{SW}^{n}(\alpha)$, and let $X \sim \mathrm{GUE}_{d}^{0}$. Then as $n \rightarrow \infty$,

$$
\left(\frac{\lambda_{1}-n / d}{\sqrt{n / d}}, \ldots, \frac{\lambda_{d}-n / d}{\sqrt{n / d}}\right) \rightarrow\left(\operatorname{eig}_{1}(X), \ldots, \operatorname{eig}_{d}(X)\right)
$$

in distribution.

Using Fact 1.23, we expect that for a typical $\lambda$,

$$
\lambda_{1} \approx \frac{n}{d}+2 \sqrt{n}, \quad \lambda_{d} \approx \frac{n}{d}-2 \sqrt{n}
$$

and that the remaining $\lambda_{i}$ 's interpolate between these two values. (Let us also mention a line of work that has considered the case of uniform $\alpha$ in the nonasymptotic setting. Here, rather than fixing $d$ and letting $n$ tend towards infinity, $d$ is allowed to grow to infinity while $n$ scales as $n=O\left(d^{2}\right)$. In this case, Biane [Bia01] has shown a limiting theorem for the shape of $\lambda$, and Méliot [Mél10] has characterized the fluctions of $\lambda$ around its mean with a certain Gaussian process. The paper of Ivanov and Olshanski [IO02], which proves similar results for the Plancherel distribution, serves as an excellent introduction to this area.)

In the case of general $\alpha$ - the inhomogeneous random word case - it is convenient to group the indices $\{1, \ldots, d\}$ according to the degeneracies of $\alpha$.

Notation 1.25. Suppose there are $m$ distinct values among the $\alpha_{i}$ 's, and write $\alpha^{(k)}$ for the $k$ th largest distinct value. We will block the indices as

$$
[1, d]=\left[1, d^{(1)}\right] \cup\left[d^{(1)}+1, d^{(1)}+d^{(2)}\right] \cup \cdots \cup\left[d-d^{(m)}+1, d\right],
$$

where every $\alpha_{i}$ in the $k$ th block has the value $\alpha^{(k)}$. Given a partition $\lambda$ of height $d$, we will write $\lambda_{i}^{(k)}$ for the $i$ th index in the $k$ th block, i.e. $\lambda_{i}^{(k)}=\lambda_{d_{<k}+i}$, where $d_{<k}=d^{(1)}+\cdots+d^{(k-1)}$. (We will only use this notation in this subsection; in particular, for Theorem 1.26.) 
In the inhomogeneous case, Its, Tracy, and Widom [ITW01] gave a limiting characterization for the first row $\lambda_{1}$, and Houdré and $\mathrm{Xu}$ [HX13], in a work that first appeared in 2009, extended their result to hold for the entire diagram $\lambda$. Roughly, their characterization shows that within each block, $\lambda$ acts GUE-like, as in Theorem 1.24, but across blocks $\lambda$ acts Gaussian-like, as in Theorem 1.21. We cite here a related theorem of Méliot [Mél12], which cleanly decouples these two limiting effects.

Theorem 1.26 ([MÉL12]). Let $\alpha=\left(\alpha_{1}, \ldots, \alpha_{d}\right)$ be a sorted probability distribution. Let $\boldsymbol{\lambda} \sim \mathrm{SW}^{n}(\alpha)$, let $\left(\boldsymbol{g}_{1}, \ldots, \boldsymbol{g}_{m}\right)$ be centered jointly Gaussian random variables with covariances given by $\delta_{k \ell} d^{(k)}$ $d^{(k)} d^{(\ell)} \sqrt{\alpha^{(k)} \alpha^{(\ell)}}$, and let $Y^{(k)} \sim \mathrm{GUE}_{d^{(k)}}^{0}$, for each $k \in[m]$. Then as $n \rightarrow \infty$,

$\left\{\frac{\lambda_{i}^{(k)}-\alpha^{(k)} n}{\sqrt{\alpha^{(k)} n}}\right\}_{k \in[m], i \in\left[d^{(k)}\right]} \rightarrow\left\{\frac{g_{k}}{d^{(k)}}+\operatorname{eig}_{i}\left(Y^{(k)}\right)\right\}_{k \in[m], i \in\left[d^{(k)}\right]}$ in distribution.

Note that this theorem recovers Theorem 1.21 in the case when all the $\alpha_{i}$ 's are distinct and Theorem 1.24 in the case when $\alpha$ is the uniform distribution. More generally, if we define $\lambda[k]=\lambda_{1}^{(k)}+\cdots+$ $\lambda_{d^{(k)}}^{(k)}$, then the random variables $\left(\lambda[k]-\alpha^{(k)} d^{(k)} n\right) / \sqrt{\alpha^{(k)} d^{(k)} n}$ converge to Gaussian random variables with covariance $\delta_{k \ell}-$ $\sqrt{\alpha^{(k)} d^{(k)} \alpha^{(\ell)} d^{(\ell)}}$. Hence, within blocks, $\lambda$ experiences GUE fluctuations, whereas across blocks, $\lambda$ experiences Gaussian fluctuations.

Theorem 1.26 predicts qualitatively different limiting behaviors between the cases when two $\alpha_{i}$ 's are exactly equal and when two $\alpha_{i}$ 's are unequal, even if they are close. Hence its convergence rate naturally depends on quantities like

$$
\max _{i: \alpha_{i} \neq \alpha_{i+1}}\left(\frac{1}{\alpha_{i}-\alpha_{i+1}}\right),
$$

and it is therefore not applicable in the nonasymptotic regime. Nevertheless, we have found it useful when reasoning about the Schur-Weyl distribution; in particular, by disregarding the Gaussian term in Theorem 1.26, we have our ansatz.

\subsection{Future Work}

Bavarian, Mehraban, and Wright have used the techniques in this work to study the accuracy of the empirical entropy estimator for learning the von Neumann entropy. In preliminary work [BMW16], they have shown the following result:

THEOREM 1.27. The bias of the empirical entropyestimator satisfies

$$
H(\alpha)-\frac{3 d^{2}}{2 n} \leq \underset{\lambda \sim \mathrm{SW}^{n}(\alpha)}{\mathbf{E}} H(\underline{\lambda}) \leq H(\alpha) .
$$

Furthermore, the estimator has mean absolute error

$$
\underset{\lambda \sim \mathrm{SW}^{n}(\alpha)}{\mathrm{E}}|H(\underline{\boldsymbol{\lambda}})-H(\alpha)| \leq \frac{3 d^{2}}{2 n}+\sqrt{\frac{2+\log (d+e)^{2}}{n}} .
$$

Hence, the empirical entropy is $\epsilon$-close to the true von Neumann entropy with high probability when $n=O\left(d^{2} / \epsilon+\log (d)^{2} / \epsilon^{2}\right)$.

This gives an expression similar to both the bias and the mean absolute error of the classical empirical entropy estimator [WY16].

\subsection{Organization}

Section 2 contains the preliminaries, Section 3 contains the proof of Theorem 1.13, Section 4 contains our results on the concentration of the Schur-Weyl distribution, Finally, due to space limitations, the proofs of our tomography results and lower-rows majorization theorem are deferred to the full version of the paper.

Acknowledgments. We would like to thank Mohammad Bavarian and Saeed Mehraban for allowing us to mention the entropy estimation result.

\section{PRELIMINARIES}

Please refer to Section 2 of [OW15] for many of the definitions and notations used in this paper. We will also introduce additional notation in this section, and establish some simple results.

Notation 2.1. Given a sequence $\eta=\left(\eta_{1}, \ldots, \eta_{d}\right)$ we write $\eta_{\leq k}=$ $\eta_{1}+\cdots+\eta_{k}$ and we write $\eta_{>k}=\eta_{k+1}+\cdots+\eta_{d}$.

The following observation concerning Lipschitz constants of the RSK algorithm is very similar to one made in [BL12, Proposition 2.1]:

Proposition 2.2. Suppose $w, w^{\prime} \in[d]^{n}$ differ in exactly one coordinate. Write $\lambda=\operatorname{shRSK}(w), \lambda^{\prime}=\operatorname{shRSK}\left(w^{\prime}\right)$. Then:

- $\left|\lambda_{\leq k}-\lambda_{\leq k}^{\prime}\right| \leq 1$ for every $k \in[d]$.

- $\left|\lambda_{k}-\lambda_{k}^{\prime}\right| \leq 2$ for every $k \in[d]$.

Proof. It suffices to prove the first statement; then, using the $k$ and $k-1$ cases, we get the second statement via the triangle inequality. Also, by interchanging the roles of $w$ and $w^{\prime}$, it suffices to prove $\lambda_{\leq k}-\lambda_{\leq k}^{\prime} \leq 1$. This follows from Greene's Theorem: $\lambda_{\leq k}$ is the length of the longest disjoint union $U$ of $k$ increasing subsequences in $w$. If $w^{\prime}$ is formed by changing one letter in $w^{\prime}$, we can simply delete this letter from $U$ (if it appears) and get a disjoint union of $k$ increasing subsequences in $w^{\prime}$ of length at least $\lambda_{\leq k}-1$. But Greene's Theorem implies this is a lower bound on $\lambda_{\leq k}^{\prime}$.

Remark 2.3. The bound of 2 in the second statement may be tight; e.g., $\operatorname{shRSK}(232122)=(4,1,1), \operatorname{shRSK}(233122)=(3,3,0)$.

Proposition 2.4. Let $\alpha, \alpha^{\prime}$ be probability distributions on $[d]$ and let $\lambda \sim \mathrm{SW}^{n}(\alpha), \lambda^{\prime} \sim \mathrm{SW}^{n}\left(\alpha^{\prime}\right)$. Then:

$$
\begin{aligned}
& \text { - }\left|\mathrm{E}\left[\underline{\lambda}_{\leq k}\right]-\mathrm{E}\left[\underline{\lambda}_{\leq k}^{\prime}\right]\right| \leq d_{\mathrm{TV}}\left(\alpha, \alpha^{\prime}\right) \text { for every } k \in[d] . \\
& \text { - }\left|\mathrm{E}\left[\underline{\lambda}_{k}\right]-\mathrm{E}\left[\underline{\lambda}_{k}^{\prime}\right]\right| \leq 2 d_{\mathrm{TV}}\left(\alpha, \alpha^{\prime}\right) \text { for every } k \in[d] .
\end{aligned}
$$

Proof. Again, it suffices to prove the first statement, as the second one easily follows. Write $\epsilon=d_{\mathrm{TV}}\left(\alpha, \alpha^{\prime}\right)$. Thus there is a coupling $\left(\boldsymbol{a}, \boldsymbol{a}^{\prime}\right)$ such that $\boldsymbol{a} \sim \alpha, \boldsymbol{a}^{\prime} \sim \alpha^{\prime}$, and $\operatorname{Pr}\left[\boldsymbol{a} \neq \boldsymbol{a}^{\prime}\right]=\epsilon$. Making $n$ independent draws from the coupled distribution and calling the resulting words $\left(\boldsymbol{w}, \boldsymbol{w}^{\prime}\right)$, it follows that $\mathrm{E}\left[\Delta\left(\boldsymbol{w}, \boldsymbol{w}^{\prime}\right)\right]=\epsilon n$, where $\Delta$ denotes Hamming distance. Thus repeated application of Proposition 2.2 yields $\left|\mathbf{E}\left[\lambda_{\leq k}\right]-\mathbf{E}\left[\lambda^{\prime}{ }_{\leq k}\right]\right| \leq \epsilon n$, where $\lambda=$ $\operatorname{shRSK}(\boldsymbol{w}), \boldsymbol{\lambda}^{\prime}=\operatorname{shRSK}\left(\boldsymbol{w}^{\prime}\right)$. But now $\lambda \sim \operatorname{SW}^{n}(\alpha), \lambda^{\prime} \sim \operatorname{SW}^{n}\left(\alpha^{\prime}\right)$, so the result follows after dividing through by $n$.

The following lemma, while simple, is crucial for our nonasymptotic estimates: 
LEMMA 2.5. Let $\alpha$ be a probability distribution on $[d]$ with $\alpha_{1} \geq$ $\alpha_{2} \geq \cdots \geq \alpha_{d}$. Fix $k \in[d]$. Then

$$
\underset{\lambda \sim \mathrm{SW}^{n}(\alpha)}{\mathrm{E}}\left[\lambda_{\leq k}-\alpha_{\leq k} n\right]
$$

is a nondecreasing function of $n$.

Proof. We begin by "reversing" the alphabet $[d]$, so that $1>$ $2>\cdots>d$; recall that this does not change the distribution of $\lambda$. Further, we will consider all $n$ simultaneously by letting $\Lambda$ be drawn from the Schur-Weyl process associated to $\boldsymbol{w} \sim \alpha^{\otimes \infty}$. Now

$\mathrm{E}\left[\Lambda_{\leq k}^{(n)}\right]=\sum_{t=1}^{n} \operatorname{Pr}\left[t^{\text {th }}\right.$ letter of $w$ creates a box in the first $k$ rows $]$.

If $\boldsymbol{w}_{t} \in[k]$ (i.e., it is among the $k$ largest letters), then it will surely create a box in the first $k$ rows. Since this occurs with probability $\alpha_{\leq k}$ for each $t$, we conclude that

$$
\begin{aligned}
& \mathrm{E}\left[\Lambda_{\leq k}^{(n)}-\alpha_{\leq k} n\right] \\
&=\sum_{t=1}^{n} \operatorname{Pr}\left[\boldsymbol{w}_{t}>k \text { and it creates a box in the first } k \text { rows }\right]
\end{aligned}
$$

This is evidently a nondecreasing function of $n$.

\subsection{Distance Measures}

Definition 2.6. Let $\alpha, \beta \in \mathbb{R}^{d}$ be probability distributions. Then the truncated Hellinger-squared distance is given by

$$
d_{\mathrm{H}^{2}}^{(k)}(\alpha, \beta)=d_{\mathrm{H}}^{(k)}(\alpha, \beta)^{2}=\sum_{i=1}^{k}\left(\sqrt{\alpha_{i}}-\sqrt{\beta_{i}}\right)^{2},
$$

and the $k=d$ case gives $d_{\mathrm{H}}(\alpha, \beta)=d_{\mathrm{H}}^{(d)}(\alpha, \beta)$ and $d_{\mathrm{H}^{2}}(\alpha, \beta)=$ $d_{\mathrm{H}^{2}}^{(d)}(\alpha, \beta)$. The truncated chi-squared divergence is given by

$$
d_{\chi^{2}}^{(k)}(\alpha, \beta)=\sum_{i=1}^{k} \beta_{i}\left(\frac{\alpha_{i}}{\beta_{i}}-1\right)^{2}
$$

and the $k=d$ case gives $d_{\chi^{2}}(\alpha, \beta)=d_{\chi^{2}}^{(d)}(\alpha, \beta)$. The truncated $\ell_{2}^{2}$ distance is given by

$$
d_{\ell_{2}^{2}}^{(k)}(\alpha, \beta)=\sum_{i=1}^{k}\left(\alpha_{i}-\beta_{i}\right)^{2}
$$

and the $k=d$ case gives $d_{\ell_{2}^{2}}(\alpha, \beta)=d_{\ell_{2}^{2}}^{(d)}(\alpha, \beta)$. Finally, the KullbackLiebler $(K L)$ divergence is given by

$$
d_{\mathrm{KL}}(\alpha, \beta)=\sum_{i=1}^{d} \alpha_{i} \ln \left(\frac{\alpha_{i}}{\beta_{i}}\right) .
$$

Proposition 2.7. These distance measures are related as

$$
\begin{aligned}
d_{\mathrm{H}^{2}}(\alpha, \beta) \leq d_{\chi^{2}}(\alpha, \beta), & d_{\mathrm{H}^{2}}^{(k)}(\alpha, \beta) \leq d_{\chi^{2}}^{(k)}(\alpha, \beta), \\
& \text { and } d_{\mathrm{KL}}(\alpha, \beta) \leq d_{\chi^{2}}(\alpha, \beta) .
\end{aligned}
$$

Proof. For the first and second inequalities, the bound follows term-by-term:

$$
\begin{aligned}
\left(\sqrt{\alpha_{i}}-\sqrt{\beta_{i}}\right)^{2} & =\beta_{i}\left(\sqrt{\frac{\alpha_{i}}{\beta_{i}}}-1\right)^{2} \\
\leq & \beta_{i}\left(\sqrt{\frac{\alpha_{i}}{\beta_{i}}}-1\right)^{2}\left(\sqrt{\frac{\alpha_{i}}{\beta_{i}}}+1\right)^{2}=\beta_{i}\left(\frac{\alpha_{i}}{\beta_{i}}-1\right)^{2} .
\end{aligned}
$$

On the other hand, the third inequality is proven considering the whole sum at once:

$$
\sum_{i=1}^{d} \alpha_{i} \ln \left(\frac{\alpha_{i}}{\beta_{i}}\right) \leq \sum_{i=1}^{d} \alpha_{i}\left(\frac{\alpha_{i}}{\beta_{i}}-1\right)=\sum_{i=1}^{d} \frac{\alpha_{i}^{2}}{\beta_{i}}-1,
$$

where the inequality uses $\ln (x) \leq x-1$ for all $x>0$, and it can be checked that the right-most quantity is equal to $d_{\chi^{2}}(\alpha, \beta)$.

Definition 2.8. Let $\rho, \sigma$ be density matrices. The fidelity is given by $F(\rho, \sigma)=\|\sqrt{\rho} \sqrt{\sigma}\|_{1}$. Related is the affinity, given by $A(\rho, \sigma)=$ $\operatorname{tr}(\sqrt{\rho} \sqrt{\sigma})$. Finally, the quantum Hellinger-squared distance is given by

$$
d_{\mathrm{H}^{2}}(\rho, \sigma)=d_{\mathrm{H}}(\rho, \sigma)^{2}=\operatorname{tr}\left((\sqrt{\rho}-\sqrt{\sigma})^{2}\right)=2-2 A(\rho, \sigma) .^{2}
$$

By definition, $d_{\mathrm{H}}(\rho, \sigma)=\|\sqrt{\rho}-\sqrt{\sigma}\|_{F}$, and hence it satisfies the triangle inequality.

Proposition 2.9. These distance measures are related as $F(\rho, \sigma)^{2} \leq$ $A(\rho, \sigma) \leq F(\rho, \sigma)$. As a result, if $d_{\mathrm{H}^{2}}(\rho, \sigma)=\epsilon$, then $1-\epsilon / 2 \leq$ $F(\rho, \sigma)$.

Proof. The upper bound $A(\rho, \sigma) \leq F(\rho, \sigma)$ is immediate, as $\operatorname{tr}(M) \leq\|M\|_{1}$ for any matrix $M$. As for the lower bound, it follows from Equations (28) and (32) from [ANSV08].

As a result, fidelity and affinity are essentially equivalent in the " $1-\epsilon$ " regime, and further it suffices to upper bound the Hellingersquared distance if we want to lower bound the fidelity. For other properties of the affinity, see [LZ04, MM15]. Though we only ever apply the fidelity to density matrices, we will sometimes apply the affinity to arbitrary positive semidefinite matrices, as in Theorem 1.19.

\section{BOUNDING THE EXCESS}

In this section we will study the quantity

$$
E_{k}^{(n)}(\alpha)=\underset{\lambda \sim \mathrm{SW}^{n}(\alpha)}{\mathrm{E}}\left[\lambda_{\leq k}-\alpha_{\leq k} n\right],
$$

where $\alpha$ is a sorted probability distribution $[d]$, and $k \in[d]$. One way to think about this quantity is as

$$
\underset{\boldsymbol{w} \sim \alpha^{\otimes n}}{\mathbf{E}}\left[\boldsymbol{\lambda}_{\leq k}-\boldsymbol{h}_{\leq k}\right],
$$

where $\boldsymbol{\lambda}=\operatorname{shRSK}(\boldsymbol{w})$ and $\boldsymbol{h}=\operatorname{Histogram}(\boldsymbol{w})$, i.e. $\boldsymbol{h}_{i}$ is the number of $i$ 's in $\boldsymbol{w}$. By Greene's Theorem we know that $\lambda>\boldsymbol{h}$ always; thus $E_{k}^{(n)}(\alpha) \geq 0$. We are therefore concerned with upper bounds, trying to quantify how "top-heavy" $\lambda$ is on average (compared to a typical $\boldsymbol{h}$ ).

As we will see (and as implicitly shown in work of Its, Tracy, and Widom [ITW01]), the distribution of $\lambda \sim \mathrm{SW}^{n}(\alpha)$ is very close to

\footnotetext{
${ }^{2}$ We note that the quantum and classical Hellinger-squared distance are often defined with factors of $\frac{1}{2}$ in front. We have omitted them for simplicity.
} 
that of a certain modification of the multinomial distribution that favors top-heavy Young diagrams.

Definition 3.1. For a sorted probability distribution $\alpha$ with all $\alpha_{i}$ 's distinct, define the function $\phi_{n, \alpha}: \mathbb{R}^{n} \rightarrow \mathbb{R}$ by

$$
\phi_{n, \alpha}(h)=1+\sum_{1 \leq i<j \leq d} \frac{\alpha_{j}}{\alpha_{i}-\alpha_{j}}\left(\frac{h_{i}}{\alpha_{i} n}-\frac{h_{j}}{\alpha_{j} n}\right) .
$$

For $\boldsymbol{h} \sim \operatorname{Mult}(n, \alpha)$ we have $\mathbf{E}\left[\boldsymbol{h}_{\ell}\right]=\alpha_{\ell} n$; thus $\mathbf{E}\left[\phi_{n, \alpha}(\boldsymbol{h})\right]=1$. We may therefore think of $\phi_{n, \alpha}(h)$ as a relative density with respect to the $\operatorname{Mult}(n, \alpha)$ distribution - except for the fact that we don't necessarily have $\phi_{n, \alpha}(h) \geq 0$ always. That will not bother us, though; we will only ever compute expectations relative to this density.

Definition 3.2. We define the modified $\alpha$-multinomial (signed) distribution on size- $n, d$-letter histograms $h$ by $\phi_{n, \alpha}(h) M_{n, \alpha}(h)$, where $M_{n, \alpha}(h)$ is the probability of $h$ under $\operatorname{Mult}(n, \alpha)$. We use the notation

$$
\begin{aligned}
\underset{\boldsymbol{h} \sim \operatorname{ModMult}(n, \alpha)}{\mathbf{E}}[F(\boldsymbol{h})] & =\sum_{h} \phi_{n, \alpha}(h) M_{n, \alpha}(h) F(h) \\
& =\underset{\boldsymbol{h} \sim \operatorname{Mult}(n, \alpha)}{\mathbf{E}}\left[\phi_{n, \alpha}(\boldsymbol{h}) F(\boldsymbol{h})\right] .
\end{aligned}
$$

As we will see in the proof of Theorem 3.7 below, for each $\lambda \vdash n$,

$$
\text { “ } \operatorname{Pr}_{\lambda \sim \mathrm{SW}^{n}(\alpha)}[\lambda=\lambda] \approx \operatorname{Pr}_{\boldsymbol{h} \sim \operatorname{ModMult}(n, \alpha)}[\boldsymbol{h}=\lambda] ” .
$$

Remark 3.3. The modified $\alpha$-multinomial distribution is only defined when $\alpha_{1}>\alpha_{2}>\cdots>\alpha_{d}$. Note that under this condition, a draw $\boldsymbol{h} \sim \operatorname{Mult}(n, \alpha)$ will have $\boldsymbol{h}_{1} \geq \boldsymbol{h}_{2} \geq \cdots \geq \boldsymbol{h}_{d}$ with "very high" probability, and thus be a genuine partition $\boldsymbol{h} \vdash n$. (The "very high" here is only when $n$ is sufficiently large compared to all of the $\frac{1}{\alpha_{k}-\alpha_{k+1}}$ values, though.)

The approximation (3) is consistent with the ansatz. One can see from the $\left(\frac{\lambda_{i}}{\alpha_{i} n}-\frac{\lambda_{j}}{\alpha_{j} n}\right)$ part of the formula for $\phi_{n, \alpha}(\lambda)$ that it emphasizes $\lambda$ 's that are "top-heavy". That is, it gives more probability to $\lambda$ 's that exceed their multinomial-expectation at low indices and fall short of their multinomial-expectation at high indices. Furthermore, one can see from the $\frac{\alpha_{j}}{\alpha_{i}-\alpha_{j}}$ part of the formula that this effect becomes more pronounced when two or more $\alpha_{\ell}$ 's tend toward equality.

The utility of (3) is that we can compute certain expectations under the modified multinomial distribution easily and exactly, since it has a simple formula. Of course, we have to concern ourselves with the approximation in (3); in fact, the error can be quite unpleasant in that it depends on $d$, and even worse, on the gaps $\alpha_{k}-\alpha_{k+1}$ Nonetheless, when it comes to using (3) to estimate $E_{k}^{(n)}(\alpha)$, we will see that the increasing property (Lemma 2.5 ) will let us evade the approximation error. Toward that end, let us make a definition and some calculations:

Notation 3.4. For any sorted probability distribution $\alpha$ on $[d]$ and any $k \in[d]$ we write

$$
\operatorname{Excess}_{k}(\alpha)=\sum_{i \leq k<j} \frac{\alpha_{j}}{\alpha_{i}-\alpha_{j}} .
$$

Remark 3.5. We have $\operatorname{Excess}_{k}(\alpha)=0$ if $k=d$, and otherwise Excess $_{k}(\alpha)$ is continuous away from $\alpha_{k}=\alpha_{k+1}$, where it blows up to $\infty$. We also have the following trivial bound, which is useful if the gap $\alpha_{k}-\alpha_{k+1}$ is large:

$$
\operatorname{Excess}_{k}(\alpha) \leq k \alpha_{>k} /\left(\alpha_{k}-\alpha_{k+1}\right) .
$$

Although their proof was a little more elaborate, Its, Tracy, and Widom [ITW01] proved the following result in the special case of $k=1$ :

Proposition 3.6. If $\alpha$ is a sorted probability distribution on $[d]$ with all $\alpha_{i}$ 's distinct, then

$$
\underset{\lambda \sim \operatorname{ModMult}(n, \alpha)}{\mathrm{E}}\left[E_{k}^{(n)}(\alpha)\right]=\operatorname{Excess}_{k}(\alpha) .
$$

Proof. By definition we have

$$
\underset{\lambda \sim \operatorname{ModMult}(n, \alpha)}{\mathbf{E}}\left[\boldsymbol{\lambda}_{k}-\alpha_{k} n\right]=\underset{\boldsymbol{h} \sim \operatorname{Mult}(n, \alpha)}{\mathbf{E}}\left[\phi_{n, \alpha}(\boldsymbol{h})\left(\boldsymbol{h}_{k}-\alpha_{k} n\right)\right] .
$$

It's convenient to write

$$
\phi_{n, \alpha}(h)=1+\sum_{1 \leq i<j \leq d} \frac{\alpha_{j}}{\alpha_{i}-\alpha_{j}}\left(\frac{h_{i}-\alpha_{i} n}{\alpha_{i} n}-\frac{h_{j}-\alpha_{j} n}{\alpha_{j} n}\right) .
$$

Then using the fact that for $\boldsymbol{h} \sim \operatorname{Mult}(n, \alpha)$ we have $\mathbf{E}\left[\boldsymbol{h}_{k}\right]=\alpha_{k} n$, $\operatorname{Var}\left[\boldsymbol{h}_{k}\right]=\alpha_{k}\left(1-\alpha_{k}\right) n, \operatorname{Cov}\left[\boldsymbol{h}_{k}, \boldsymbol{h}_{\ell}\right]=-\alpha_{k} \alpha_{\ell} n$, we easily obtain:

$$
\underset{\lambda \sim \operatorname{ModMult}(n, \alpha)}{\mathrm{E}}\left[\lambda_{k}-\alpha_{k} n\right]=\sum_{j>k} \frac{\alpha_{j}}{\alpha_{k}-\alpha_{j}}-\sum_{i<k} \frac{\alpha_{k}}{\alpha_{i}-\alpha_{k}} .
$$

The result follows.

We now come to the main result of this section:

Theorem 3.7. Let $\alpha$ be a sorted probability distribution on $[d]$ and let $k \in[d]$. Then for all $n \in \mathbb{N}$,

$$
\underset{\lambda \sim \mathrm{SW}^{n}(\alpha)}{\mathrm{E}}\left[\lambda_{\leq k}-\alpha_{\leq k} n\right] \leq \operatorname{Excess}_{k}(\alpha) .
$$

Furthermore, using the notation $E_{k}^{(n)}(\alpha)$ for the left-hand side, it holds that $E_{k}^{(n)}(\alpha) \nearrow \operatorname{Excess}_{k}(\alpha)$ as $n \rightarrow \infty$ provided that all $\alpha_{i}$ 's are distinct.

Remark 3.8. We expect that $E_{k}^{(n)}(\alpha) \nearrow \operatorname{Excess}_{k}(\alpha)$ for all $\alpha$; however we did not prove this.

Proof. Lemma 2.5 tells us that $E_{k}^{(n)}(\alpha)$ is nondecreasing in $n$ for all $\alpha$ and $k$; thus $E_{k}^{(n)}(\alpha) \nearrow L_{k}(\alpha)$ for some $L_{k}(\alpha) \in \mathbb{R} \cup\{\infty\}$. The main claim that will complete the proof is the following (the $k=1$ case of which was proven in [ITW01]):

Claim 3.9. For fixed $\alpha$ and $k$,

$E_{k}^{(n)}(\alpha)=\operatorname{Excess}_{k}(\alpha) \pm O(1 / \sqrt{n}) \quad$ provided the $\alpha_{i}$ 's are distinct, where the constant hidden in the $O(\cdot)$ may depend on $\alpha$ in an arbitrary way.

This claim establishes $L_{k}(\alpha)=\operatorname{Excess}_{k}(\alpha)$ whenever the $\alpha_{i}$ 's are all distinct. It remains to observe that when the $\alpha_{i}$ 's are not all distinct, $L_{k}(\alpha)>\operatorname{Excess}_{k}(\alpha)$ is impossible; this is because Excess $_{k}(\alpha)-E_{k}^{(n)}(\alpha)$ is a continuous function of $\alpha$ for each fixed $n$ (unless $\alpha_{k}=\alpha_{k+1}$, but in this case $\operatorname{Excess}_{k}(\alpha)=\infty$ and there is nothing to prove). 
We now focus on proving Claim 3.9, following the analysis in [ITW01]. We emphasize that the $\alpha_{i}$ 's are now assumed distinct, and the constants hidden in all subsequent $O(\cdot)$ notation may well depend on the $\alpha_{i}$ 's.

As computed in [ITW01, top of p. 255], for each $\lambda \vdash n$ we have

$$
\begin{aligned}
& \operatorname{Pr}_{\lambda \sim \mathrm{SW}^{n}(\alpha)}[\lambda=\lambda] \\
& =\left(1+\frac{1}{\sqrt{n}}\left(\sum_{1 \leq i<j \leq d} \sqrt{\frac{\alpha_{j}}{\alpha_{i}}} \frac{\sqrt{\alpha_{j}} \xi_{i}-\sqrt{\alpha_{i}} \xi_{j}}{\alpha_{i}-\alpha_{j}}\right) \pm O\left(\frac{1}{n}\right)\right) M_{n, \alpha}(\lambda) \\
& \pm e^{-\Omega(n)}
\end{aligned}
$$

where $\xi_{\ell}=\left(\lambda_{\ell}-\alpha_{\ell} n\right) / \sqrt{\alpha_{\ell} n}$. If we now simply substitute in the definition of $\xi_{\ell}$ and do some simple arithmetic, we indeed get the following precise form of (3):

$$
\underset{\lambda \sim \mathrm{SW}^{n}(\alpha)}{\operatorname{Pr}}[\lambda=\lambda]=\phi_{n, \alpha}(\lambda) M_{n, \alpha}(\lambda) \pm O\left(\frac{M_{n, \alpha}(\lambda)}{n}\right) \pm e^{-\Omega(n)} .
$$

Given this, let $F$ be any functional on partitions of $n$ that is subexponentially bounded in $n$ (meaning $|F(\lambda)| \leq e^{o(n)}$ for all $\lambda \vdash$ $n)$. Then

$$
\begin{aligned}
\underset{\lambda \sim \mathrm{SW}^{n}(\alpha)}{\mathbf{E}}[F(\lambda)]=\underset{\lambda \sim \operatorname{ModMult}(n, \alpha)}{\mathbf{E}}\left[\mathbf{1}_{\{\lambda \text { is sorted }\}} \cdot F(\lambda)\right] \\
\pm O\left(\frac{1}{n}\right) \cdot \underset{\lambda \sim \operatorname{Mult}(n, \alpha)}{\mathrm{E}}\left[\mathbf{1}_{\{\boldsymbol{\lambda} \text { is sorted }\}} \cdot|F(\lambda)|\right] \pm e^{-\Omega(n)},
\end{aligned}
$$

where in the final error $e^{\Omega(n)}$ we used the subexponential bound on $|F(\lambda)|$ and also absorbed a factor of $e^{O(\sqrt{n})}$, the number of partitions of $n$. We can further simplify this: Using $\alpha_{1}>\alpha_{2}>\cdots>\alpha_{d}$, an easy Chernoff/union bound gives that

$$
\operatorname{Pr}_{\lambda \sim \operatorname{Mult}(n, \alpha)}[\lambda \text { is not sorted }] \leq e^{-\Omega(n)}
$$

(where certainly the constant in the $\Omega(\cdot)$ depends on all the gaps $\left.\alpha_{\ell}-\alpha_{\ell+1}\right)$. Thus

$$
\begin{aligned}
& \left|\underset{\lambda \sim \operatorname{ModMult}(n, \alpha)}{\mathbf{E}}\left[\mathbf{1}_{\{\lambda \text { is unsorted }\}} \cdot F(\lambda)\right]\right| \\
& \quad=\left|\underset{\lambda \sim \operatorname{Mult}(n, \alpha)}{\mathbf{E}}\left[\mathbf{1}_{\{\lambda \text { is unsorted }\}} \cdot \phi_{n, \alpha}(\lambda) F(\lambda)\right]\right| \\
& \leq \sqrt{\underset{\lambda \sim \operatorname{Mult}(n, \alpha)}{\mathbf{E}}\left[\mathbf{1}_{\{\lambda \text { is unsorted }\}}^{2}\right]} \sqrt{\phi_{n, \alpha}(\lambda)^{2} F(\lambda)^{2}} \leq e^{-\Omega(n)},
\end{aligned}
$$

where we used (7), the subexponential bound on $F$, and $\phi_{n, \alpha}(\lambda) \leq$ $O(1)$. A similar but simpler analysis applies to the first middle term in (8), and we conclude the following attractive form of (3) for subexponentially-bounded $F$ :

$$
\begin{aligned}
& \underset{\lambda \sim \mathrm{SW}^{n}(\alpha)}{\mathbf{E}}[F(\boldsymbol{\lambda})] \\
& =\underset{\lambda \sim \operatorname{ModMult}(n, \alpha)}{\mathbf{E}}[F(\boldsymbol{\lambda})] \quad \pm O\left(\frac{1}{n}\right) \cdot \underset{\lambda \sim \operatorname{Mult}(n, \alpha)}{\mathbf{E}}[|F(\lambda)|] \pm e^{-\Omega(n)} .
\end{aligned}
$$

Finally, Claim (3.9) now follows from Proposition 3.6, together with the fact that for $\lambda \sim \operatorname{Mult}(n, \alpha)$,

$$
\begin{aligned}
& \mathrm{E}\left[\left|E_{k}^{(n)}(\lambda)\right|\right] \leq \sum_{i=1}^{k} \sqrt{\mathrm{E}\left[\left(\lambda_{i}-\alpha_{i} n\right)^{2}\right]} \\
& =\sum_{i=1}^{k} \operatorname{stddev}\left[\lambda_{i}\right]=\sum_{i=1}^{k} \sqrt{n} \sqrt{\alpha_{i}\left(1-\alpha_{i}\right)}=O(\sqrt{n}) .
\end{aligned}
$$

\section{CONVERGENCE OF THE SCHUR-WEYL DISTRIBUTION}

In this section, we derive consequences of Theorem 1.13 and Theorem 1.12. To begin, it will help to define two restrictions of a word $w$.

Notation 4.1. Let $w \in[d]^{n}$ and let $\lambda=\operatorname{shRSK}(w)$. We use boldface $\boldsymbol{w}$ if $\boldsymbol{w} \sim \alpha^{\otimes n}$, in which case $\lambda \sim \mathrm{SW}^{n}(\alpha)$.

- Write $w^{(k . .)}$ for the string formed from $w$ by deleting all letters smaller than $k$, and let $\lambda^{\left(k_{.} .\right)}=\operatorname{shRSK}\left(w^{\left(k_{. .}\right)}\right)$. Then the random variable $\lambda^{\left(k_{.}\right)}$is distributed as $\operatorname{SW}^{\ell}(\alpha[k:])$, where $\boldsymbol{\ell} \sim \operatorname{Binomial}\left(n, \alpha_{\geq k}\right)$ and $\underline{\alpha[k:]}=\left(\alpha_{i} / \alpha_{\geq k}\right)_{i=k}^{\bar{d}}$.

- Write $w^{(. . k)}$ for the string formed from $w$ by deleting all letters larger than $k$, and let $\lambda^{(. . k)}=\operatorname{shRSK}\left(w^{(. . k)}\right)$. Note that if $(P, Q)=\operatorname{RSK}(w)$, then $\lambda^{(. . k)}$ is the shape of the diagram formed by deleting all boxes containing letters larger than $k$ from $P$, and hence $\lambda_{i}^{(. . k)} \leq \lambda_{i}$ for all $i$. Then the random variable $\lambda^{(. . k)}$ is distributed as $\operatorname{SW}^{\boldsymbol{m}}(\alpha[: k])$, where $\boldsymbol{m} \sim \operatorname{Binomial}\left(n, \alpha_{\leq k}\right)$ and $\underline{\alpha[: k]}=\left(\alpha_{i} / \alpha_{\leq k}\right)_{i=1}^{k}$.

We will mainly use the following weaker version of Theorem ??.

TheOREM 4.2. Let $\lambda[k:]$ denote the Young diagram formed by rows $k, k+1, k+2, \ldots$ of $\lambda$. Then $\lambda^{(k . .)} \unrhd_{w} \lambda[k:]$.

Proof. This follows by applying Theorem 1.12 to $w$ and noting that the string $\bar{w}$ in that theorem is a substring of $w^{(k . .)}$. Hence weak majorization holds trivially.

\subsection{Bounds on the First and Last Rows}

Theorem 4.3. Let $\alpha \in \mathbb{R}^{d}$ be a sorted probability distribution. Then

$$
\underset{\lambda \sim \mathrm{SW}^{n}(\alpha)}{\mathrm{E}} \lambda_{1} \leq \alpha_{1} n+2 \sqrt{n}
$$

Proof. Write $g=1 / \sqrt{n}$. We assume that $\alpha_{1}+2 g \leq 1$, as otherwise the theorem is vacuously true. Let $\beta \in \mathbb{R}^{d}$ be a sorted probability distribution for which $\beta_{1}=\alpha_{1}+g, \beta_{2} \leq \alpha_{2}$, and $\beta>\alpha$. Then

$$
\begin{aligned}
\underset{\lambda \sim \mathrm{SW}^{n}(\alpha)}{\mathbf{E}} \lambda_{1} \leq \underset{\mu \sim \mathrm{SW}^{n}(\beta)}{\mathrm{E}} \boldsymbol{\mu}_{1} \leq \beta_{1} n+\sum_{j>1} \frac{\beta_{j}}{\beta_{1}-\beta_{j}} \\
\quad \leq \beta_{1} n+\frac{1}{g}=\alpha_{1} n+n g+\frac{1}{g}=\alpha_{1} n+2 \sqrt{n},
\end{aligned}
$$

where the first step is by Theorem 1.14 and the second is by Theorem 1.13. 
Theorem 4.4. Let $\alpha \in \mathbb{R}^{d}$ be a sorted probability distribution. Then

$$
\underset{\lambda \sim \mathrm{SW}^{n}(\alpha)}{\mathrm{E}} \lambda_{d} \geq \alpha_{d} n-2 \sqrt{\alpha_{d} d n}
$$

Proof. Write $g=\sqrt{\alpha_{d} d / n}$. We assume that $\alpha_{d}-2 g \geq 0$, as otherwise the theorem is vacuously true. Let $\beta \in \mathbb{R}^{d}$ be a sorted probability distribution for which $\beta_{d}=\alpha_{d}-g, \beta_{d-1} \geq \alpha_{d-1}$, and $\beta>\alpha$. Then

$$
\begin{gathered}
\underset{\lambda \sim \mathrm{SW}^{n}(\alpha)}{\mathbf{E}}\left[\lambda_{1}+\cdots+\lambda_{d-1}\right] \leq \underset{\mu \sim \mathrm{SW}^{n}(\beta)}{\mathbf{E}}\left[\boldsymbol{\mu}_{1}+\cdots+\mu_{d-1}\right] \\
\leq \beta_{1} n+\cdots+\beta_{d-1} n+\sum_{i<d} \frac{\beta_{d}}{\beta_{i}-\beta_{d}} \\
\leq \beta_{1} n+\cdots+\beta_{d-1} n+\frac{d \alpha_{d}}{g}=\alpha_{1} n+\cdots+\alpha_{d-1} n+2 \sqrt{\alpha_{d} d n}
\end{gathered}
$$

where the first inequality is by Theorem 1.14, and the second inequality is by Theorem 1.13. As $\lambda_{1}+\cdots+\lambda_{d}=n$, this implies that $\mathrm{E} \boldsymbol{\lambda}_{d} \geq \alpha_{d} n-2 \sqrt{\alpha_{d} d n}$.

We note that Theorem 1.14 can be replaced by Proposition 2.4 at just a constant-factor expense.

\subsection{Bounds for All Rows}

Theorem 4.5. Let $\alpha \in \mathbb{R}^{d}$ be a sorted probability distribution. Then

$$
\alpha_{k} n-2 \sqrt{\alpha_{k} k n} \leq \underset{\lambda \sim \mathrm{SW}^{n}(\alpha)}{\mathrm{E}} \lambda_{k} \leq \alpha_{k} n+2 \sqrt{\alpha_{\geq k} n} .
$$

Proof. For the upper bound, we use Theorem 4.2:

$$
\begin{aligned}
\underset{\lambda \sim \mathrm{SW}^{n}(\alpha)}{\mathrm{E}} \lambda_{k} \leq \underset{\boldsymbol{\ell}, \lambda^{(k . .)}}{\mathrm{E}} \lambda_{1}^{(k . .)} \leq \underset{\boldsymbol{\ell}}{\mathrm{E}}[\underline{\alpha[k:]} k+2 \sqrt{\boldsymbol{\ell}}] \\
\leq \underline{\alpha[k:]_{k}} \mathrm{E} \boldsymbol{\ell}+2 \sqrt{\mathrm{E} \boldsymbol{\ell}} \leq \alpha_{k} n+2 \sqrt{\alpha_{\geq k} n},
\end{aligned}
$$

where the second step is by Theorem 4.3 and the third is by Jensen's inequality.

For the lower bound, we use the fact that $\lambda_{k} \geq \lambda_{k}^{(. . k)}$ :

$$
\begin{array}{r}
\underset{\lambda \sim \mathrm{SW}^{n}(\alpha)}{\mathbf{E}} \lambda_{k} \geq \underset{\boldsymbol{m}, \lambda^{(. . k)}}{\mathbf{E}} \lambda_{k}^{(. . k)} \geq \underset{\boldsymbol{m}}{\mathbf{E}}\left[\frac{\alpha[: k]}{k} \boldsymbol{m}-2 \sqrt{\underline{\alpha[: k]} k k \boldsymbol{m}}\right] \\
\geq \underline{\alpha[: k]} k \mathrm{E} \boldsymbol{m}-2 \sqrt{\underline{\alpha[: k]} k k \mathbf{E} \boldsymbol{m}}=\alpha_{k} n-2 \sqrt{\alpha_{k} k n},
\end{array}
$$

where the second inequality is by Theorem 4.4, and the third is by Jensen's inequality.

Theorem 1.4 follows from the fact that $\alpha_{k} k, \alpha_{\geq k} \leq \min \left\{1, \alpha_{k} d\right\}$.

\subsection{Chi-Squared Spectrum Estimation}

Theorem 4.6. Let $\alpha \in \mathbb{R}^{d}$ be a sorted probability distribution. Then for any $k \in[d]$,

$$
\underset{\lambda \sim \mathrm{SW}^{n}(\alpha)}{\mathrm{E}} \sum_{i=k}^{d} \lambda_{i}^{2} \leq \sum_{i=k}^{d}\left(\alpha_{i} n\right)^{2}+d \alpha_{\geq k} n .
$$

Proof. When $k=1$, this statement is equivalent to Lemma 3.1 from [OW16]. Hence, we may assume $k>1$. By Theorem 4.2,

$$
\begin{gathered}
\underset{\lambda \sim \mathrm{SW}^{n}(\alpha)}{\mathbf{E}} \sum_{i=k}^{d} \lambda_{i}^{2} \leq \underset{\boldsymbol{\ell}, \boldsymbol{\lambda}^{(k . .)}}{\mathbf{E}} \sum_{i=1}^{d-k+1}\left(\lambda_{i}^{(k . .)}\right)^{2} \\
\leq \underset{\boldsymbol{\ell}}{\mathbf{E}}\left[\sum_{i=k}^{d}\left(\frac{\alpha[k:]}{i} \boldsymbol{\ell}\right)^{2}+(d-k+1) \boldsymbol{\ell}\right] \\
=\sum_{i=k}^{d}\left(\alpha_{i} n\right)^{2}+\sum_{i=k}^{d} \alpha_{i}^{2} n\left(\frac{1}{\alpha_{\geq k}}-1\right)+(d-k+1) \alpha_{\geq k} n \leq \sum_{i=k}^{d}\left(\alpha_{i} n\right)^{2}+d \alpha_{\geq k} n .
\end{gathered}
$$

Here the second inequality used Lemma 3.1 from [OW16], and the third inequality used $\alpha_{\geq k} \geq \alpha_{i}$ and $k>1$.

$$
\text { Theorem 4.7. } \underset{\lambda \sim \mathrm{SW}^{n}(\alpha)}{\mathrm{E}} d_{\chi^{2}}(\underline{\boldsymbol{\lambda}}, \alpha) \leq \frac{d^{2}}{n} \text {. }
$$

Proof. Write the expectation as

$$
\mathbf{E} d_{\chi^{2}}(\underline{\lambda}, \alpha)=\frac{1}{n^{2}} \cdot \mathbf{E} \sum_{i=1}^{k} \frac{\lambda_{i}^{2}}{\alpha_{i}}-1
$$

To upper bound the expectation, we can apply Theorem 4.6.

$$
\begin{aligned}
\mathrm{E} \sum_{i=1}^{d} \frac{\lambda_{i}^{2}}{\alpha_{i}} & =\sum_{i=1}^{d}\left(\frac{1}{\alpha_{i}}-\frac{1}{\alpha_{i-1}}\right) \cdot \mathrm{E} \sum_{j=i}^{d} \lambda_{j}^{2} \\
& \leq \sum_{i=1}^{d}\left(\frac{1}{\alpha_{i}}-\frac{1}{\alpha_{i-1}}\right) \cdot \sum_{j=i}^{d}\left(\left(\alpha_{j} n\right)^{2}+d \alpha_{j} n\right) \\
& =\sum_{j=1}^{d}\left(\left(\alpha_{j} n\right)^{2}+d \alpha_{j} n\right) \cdot \sum_{i=1}^{j}\left(\frac{1}{\alpha_{i}}-\frac{1}{\alpha_{i-1}}\right) \\
& =\sum_{j=1}^{d}\left(\left(\alpha_{j} n\right)^{2}+d \alpha_{j} n\right) \cdot \frac{1}{\alpha_{j}}=n^{2}+d^{2} n .
\end{aligned}
$$

Dividing through by $n^{2}$ and subtracting one completes the proof.

Combined with Proposition 2.7, Theorem 4.7 implies Theorem 1.7.

\subsection{Concentration Bounds}

In this section, we show that each row $\lambda_{i}$ concentrates exponentially around its mean. We do so using the method of bounded differences.

Proposition 4.8. Let $\alpha \in \mathbb{R}^{d}$ be a probability distribution. Then for any $k \in[d]$,

$$
\underset{\lambda \sim \mathrm{SW}^{n}(\alpha)}{\operatorname{Var}}\left[\lambda_{k}\right] \leq 16 n
$$

Proof. Let $\boldsymbol{w} \sim \alpha^{\otimes n}$, and consider the martingale $X^{(0)}, \ldots, X^{(n)}$ defined as

$$
X^{(i)}:=\mathrm{E}\left[\lambda_{k} \mid w_{1}, \ldots, w_{i}\right] .
$$

Note that $\boldsymbol{X}^{(0)}=\mathbf{E} \boldsymbol{\lambda}_{k}$ and $\boldsymbol{X}^{(n)}=\operatorname{shRSK}(\boldsymbol{w})_{k}$. Furthermore, by Proposition 2.2, we have that $\left|\boldsymbol{X}^{(i)}-\boldsymbol{X}^{(i-1)}\right| \leq 2$ always, for all 
$i \in[n]$. Thus, if we write $v_{k}:=\mathrm{E} \boldsymbol{\lambda}_{k}=\boldsymbol{X}^{(0)}$, then by Azuma's inequality

$$
\operatorname{Pr}\left[\left|\lambda_{k}-v_{k}\right| \geq t\right] \leq 2 \exp \left(\frac{-t^{2}}{8 n}\right)
$$

We can therefore calculate $\operatorname{Var}\left[\lambda_{k}\right]$ as

$$
\begin{aligned}
& \mathrm{E}\left(\lambda_{k}-v_{k}\right)^{2}=\int_{t=0}^{\infty} 2 t \cdot \operatorname{Pr}\left[\left|\lambda_{k}-v_{k}\right| \geq t\right] \cdot \mathrm{d} t \\
& \leq \int_{t=0}^{\infty} 4 t \exp \left(\frac{-t^{2}}{8 n}\right) \cdot \mathrm{d} t=-\left.16 n \exp \left(\frac{-t^{2}}{8 n}\right)\right|_{t=0} ^{\infty}=16 n
\end{aligned}
$$

\subsection{Truncated Spectrum Estimation}

LemmA 4.9. Let $1 \leq i \leq k \leq d$. Then

$$
\underset{\lambda \sim S W^{n}(\alpha)}{\mathbf{E}}\left(\lambda_{i}-\alpha_{i} n\right)^{2} \leq 2 \underset{\boldsymbol{m}, \lambda^{(. . k)}}{\mathrm{E}}\left(\lambda_{i}^{(. . k)}-\underline{\alpha[: k]}_{i} \boldsymbol{m}\right)^{2}+44 \alpha_{\geq i} n .
$$

Proof. Write $\mathcal{G}$ for the event that $\lambda_{i} \geq \alpha_{i} n$. Then

$$
\underset{\lambda}{\mathrm{E}}\left(\boldsymbol{\lambda}_{i}-\alpha_{i} n\right)^{2}=\underset{\lambda}{\mathrm{E}}\left[\left(\boldsymbol{\lambda}_{i}-\alpha_{i} n\right)^{2} \cdot \mathbf{1}[\mathcal{G}]\right]+\underset{\lambda}{\mathrm{E}}\left[\left(\boldsymbol{\lambda}_{i}-\alpha_{i} n\right)^{2} \cdot \mathbf{1}[\overline{\mathcal{G}}]\right] .
$$

When $\mathcal{G}$ occurs, then $\left(\lambda_{i}-\alpha_{i} n\right)^{2} \leq\left(\lambda_{1}^{(i . .)}-\alpha_{i} n\right)^{2}$. Hence

$$
\begin{aligned}
& \mathbf{E}\left[\left(\lambda_{i}-\alpha_{i} n\right)^{2} \cdot \mathbf{1}[\mathcal{G}]\right] \leq \mathrm{E}\left(\boldsymbol{\lambda}_{1}^{(i . .)}-\alpha_{i} n\right)^{2}
\end{aligned}
$$

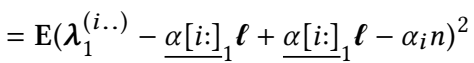

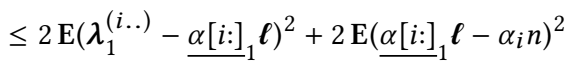

$$
\begin{aligned}
& \leq 2 \mathrm{E}\left(\lambda_{1}^{(i . .)}-\underline{\alpha[i:]}_{1} \ell\right)^{2}+\frac{2 n \alpha_{i}^{2}}{\alpha_{\geq i}},
\end{aligned}
$$

where the second inequality uses $(x+y)^{2} \leq 2 x^{2}+2 y^{2}$ for all $x, y \in \mathbb{R}$, and the third inequality is because $\boldsymbol{\ell}$ is distributed as $\operatorname{Binomial}\left(n, \alpha_{\geq i}\right)$. Given $\boldsymbol{\ell}$, define $\boldsymbol{v}=\mathrm{E}\left[\boldsymbol{\lambda}_{1}^{(i . .)} \mid \boldsymbol{\ell}\right]$. Then

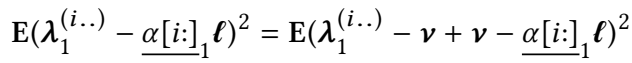

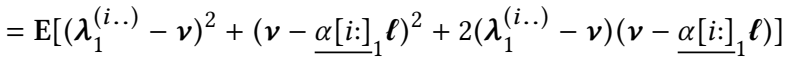

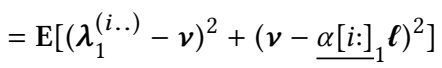

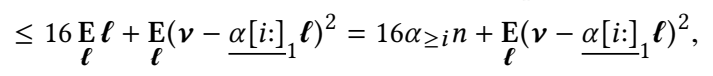

where the inequality uses Proposition 4.8. Next, we note that because $\lambda^{(i . .)}$ is distributed as $\mathrm{SW}^{\boldsymbol{\ell}}\left(\underline{\alpha[i:])}, \boldsymbol{v}\right.$ is at least $\alpha[i:]_{1} \boldsymbol{\ell}$. Hence,

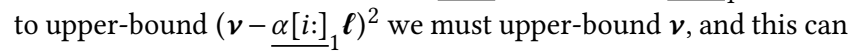

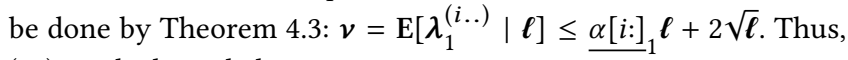
(10) can be bounded as

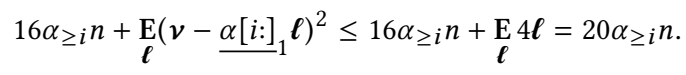

In summary, the term in (9) corresponding to $\mathcal{G}$ is at most $42 \alpha_{\geq i} n$.
As for the other term, when $\mathcal{G}$ does not occur, then $\left(\boldsymbol{\lambda}_{i}-\alpha_{i} n\right)^{2} \leq$ $\left(\lambda_{i}^{(. . k)}-\alpha_{i} n\right)^{2}$. Hence

$$
\begin{aligned}
& \mathrm{E}\left[\left(\boldsymbol{\lambda}_{i}-\alpha_{i} n\right)^{2} \cdot \mathbf{1}[\overline{\mathcal{G}}]\right] \leq \mathrm{E}\left(\boldsymbol{\lambda}_{i}^{(. . k)}-\alpha_{i} n\right)^{2} \\
& =\mathbf{E}\left(\boldsymbol{\lambda}_{i}^{(. . k)}-\underline{\alpha[: k]}_{i} \boldsymbol{m}+\underline{\alpha[: k]_{i}} \boldsymbol{m}-\alpha_{i} n\right)^{2}
\end{aligned}
$$

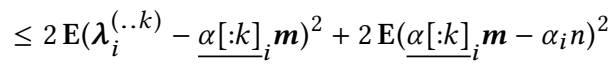

$$
\begin{aligned}
& \leq 2 \mathrm{E}\left(\boldsymbol{\lambda}_{i}^{(. . k)}-{\underline{\alpha[: k]_{i}}}_{i}\right)^{2}+\frac{2 n \alpha_{i}^{2}}{\alpha_{\leq k}},
\end{aligned}
$$

where the second inequality uses $(x+y)^{2} \leq 2 x^{2}+2 y^{2}$ for all $x, y \in \mathbb{R}$, and the third inequality is because $\boldsymbol{m}$ is distributed as $\operatorname{Binomial}\left(n, \alpha_{\leq k}\right)$. As $\alpha_{i}^{2} / \alpha_{\leq k} \leq \alpha_{\geq i}$, the proof is complete.

THEOREM 4.10. $\underset{\lambda \sim S W^{n}(\alpha)}{\mathbf{E}} d_{\ell_{2}^{2}}^{(k)}(\underline{\lambda}, \alpha) \leq \frac{46 k}{n}$.

Proof. Applying Lemma 4.9 with $j=k$ for all $i \in[k]$,

$$
\begin{aligned}
& n^{2} \mathbf{E} d_{\ell_{2}^{2}}^{(k)}(\underline{\boldsymbol{\lambda}}, \alpha)=\mathrm{E} \sum_{i=1}^{k}\left(\boldsymbol{\lambda}_{i}-\alpha_{i} n\right)^{2} \\
& \leq 2 \mathbf{E} \sum_{i=1}^{k}\left(\boldsymbol{\lambda}_{i}^{(. . k)}-\underline{\alpha[: k]} \underline{\boldsymbol{m}}^{2}+44 k n\right. \\
&= \underset{\boldsymbol{m}}{\mathbf{E}}\left[\boldsymbol{m}^{2} \underset{\lambda^{(. . k)}}{\mathbf{E}}\left\|\underline{\boldsymbol{\lambda}}^{(. . k)}-\underline{\alpha[: k]}\right\|_{2}^{2}\right]+44 k n \\
& \leq 2 \underset{\boldsymbol{m}}{\mathbf{E}}\left[\boldsymbol{m}^{2}\left(\frac{k}{\boldsymbol{m}}\right)\right]+44 k n \leq 46 k n,
\end{aligned}
$$

where the second inequality is by Theorem 1.1 of [OW16]. The theorem follows by dividing through by $n^{2}$.

THEOREM 4.11. $\underset{\lambda \sim S \mathrm{~W}^{n}(\alpha)}{\mathbf{E}} d_{\chi^{2}}^{(k)}(\underline{\boldsymbol{\lambda}}, \alpha) \leq \frac{46 k d}{n}$.

Proof. Applying Lemma 4.9 with $j=k$ for all $i \in[k]$,

$$
\begin{aligned}
& n^{2} \mathrm{E} d_{\chi^{2}}^{(k)}(\underline{\boldsymbol{\lambda}}, \alpha)=\mathrm{E} \sum_{i=1}^{k} \frac{1}{\alpha_{i}}\left(\lambda_{i}-\alpha_{i} n\right)^{2} \\
& \leq 2 \mathrm{E} \sum_{i=1}^{k} \frac{1}{\alpha_{i}}\left(\boldsymbol{\lambda}_{i}^{(. . k)}-\underline{\alpha[: k]} \underline{\boldsymbol{m}}^{2}+\sum_{i=1}^{k} \frac{44 \alpha_{\geq i} n}{\alpha_{i}}\right. \\
& \leq 2 \underset{\boldsymbol{m}}{\mathrm{E}}\left[\frac{\boldsymbol{m}^{2}}{\alpha_{\leq k}} \underset{\lambda^{(. . k)}}{\mathrm{E}} d_{\chi^{2}}\left(\underline{\boldsymbol{\lambda}^{(. . k)}}, \underline{\alpha[: k])}\right]+44 k d n\right. \\
& \quad \leq 2 \underset{\boldsymbol{m}}{\mathbf{E}}\left[\frac{\boldsymbol{m}^{2}}{\alpha_{\leq k}} \cdot \frac{k^{2}}{\boldsymbol{m}}\right]+44 k d n=2 k^{2} n+44 k d n,
\end{aligned}
$$

where the second inequality is because $\alpha_{\geq i} \leq \alpha_{i} d$, and the third inequality is by Theorem 4.7. The theorem follows from $k \leq d$ and by dividing through by $n^{2}$.

\subsection{Mean Squared Error}

THEOREM 4.12. $\underset{\lambda \sim \mathrm{SW}^{n}(\alpha)}{\mathrm{E}}\left(\lambda_{k}-\alpha_{k} n\right)^{2} \leq 42 \alpha_{k} k n+42 \alpha_{\geq k} n$.

Proof. Following the proof of Lemma 4.9 for $i=k$, we have that

$$
\underset{\lambda}{\mathrm{E}}\left(\boldsymbol{\lambda}_{k}-\alpha_{i} n\right)^{2} \leq \mathrm{E}\left[\left(\lambda_{k}^{(. . k)}-\alpha_{k} n\right)^{2} \cdot \mathbf{1}[\overline{\mathcal{G}}]\right]+42 \alpha_{\geq k} n,
$$


where $\mathcal{G}$ is the event that $\lambda_{k} \geq \alpha_{i} n$. Now we borrow a step from the proof of Lemma 5.1 in [OW16]. Because it has support size $k$, $\alpha[: k]$ can be expressed as a mixture

$$
\underline{\alpha[: k]}=p_{1} \cdot \mathcal{D}_{1}+p_{2} \cdot \mathcal{D}_{2}
$$

of a certain distribution $\mathcal{D}_{1}$ supported on $[k-1]$ and the uniform distribution $\mathcal{D}_{2}$ on $[k]$. It can be checked that $p_{2}=\alpha[: k]_{k} k$. We may therefore think of a draw $\lambda^{(. . k)}$ from $\mathrm{SW}^{\boldsymbol{m}}(\alpha[: k])$ occurring as follows. First, $[\boldsymbol{m}]$ is partitioned into two subsets $\overline{\boldsymbol{I}}_{1}, \boldsymbol{I}_{2}$ by including each $i \in[\boldsymbol{m}]$ into $\boldsymbol{I}_{j}$ independently with probability $p_{j}$. Next we draw strings $\boldsymbol{w}^{(j)} \sim \mathcal{D}_{j}^{\otimes \boldsymbol{I}_{j}}$ independently for $j \in$ [2]. Finally, we let $\boldsymbol{w}^{(. . k)}=\left(\boldsymbol{w}^{(1)}, \boldsymbol{w}^{(2)}\right) \in[d]^{n}$ be the natural composite string and define $\lambda^{(. . k)}=\operatorname{shRSK}\left(\boldsymbol{w}^{(. . k)}\right)$. Let us also write $\lambda^{(j)}=\operatorname{shRSK}\left(\lambda^{(j)}\right)$ for $j \in[2]$. We now claim that

$$
\sum_{i=1}^{z} \lambda_{i}^{(. . k)} \leq \sum_{i=1}^{z} \lambda_{i}^{(1)}+\sum_{i=1}^{z} \lambda_{i}^{(2)}
$$

always holds. Indeed, this follows from Greene's Theorem: the left-hand side is $|\boldsymbol{s}|$, where $s \in[d]^{n}$ is a maximum-length disjoint union of $z$ increasing subsequences in $\boldsymbol{w}$; the projection of $\boldsymbol{s}^{(j)}$ onto coordinates $I_{j}$ is a disjoint union of $z$ increasing subsequences in $\boldsymbol{w}^{(j)}$ and hence the right-hand side is at least $\left|\boldsymbol{s}^{(1)}\right|+\left|\boldsymbol{s}^{(2)}\right|=|\boldsymbol{s}|$.

Applying (13) in the $z=k-1$ case, and using the facts that (i) $\left|\lambda^{(. . k)}\right|=\left|\lambda^{(1)}\right|+\left|\lambda^{(2)}\right|$, and (ii) $\lambda^{(1)}$ has height at most $k-1$, we see that $\lambda_{k}^{(2)} \leq \lambda_{k}^{(. . k)}$. Hence

$$
\mathbf{E}\left[\left(\boldsymbol{\lambda}_{k}^{(. . k)}-\alpha_{k} n\right)^{2} \cdot \mathbf{1}[\overline{\mathcal{G}}]\right] \leq \mathbf{E}\left(\boldsymbol{\lambda}_{k}^{(2)}-\alpha_{k} n\right)^{2}=\underset{\boldsymbol{m}, \boldsymbol{u}, \boldsymbol{\mu}}{\mathbf{E}}\left(\boldsymbol{\mu}_{k}-\alpha_{k} n\right)^{2},
$$

where $\boldsymbol{u} \sim \operatorname{Binomial}\left(\boldsymbol{m}, p_{2}\right)$ and $\boldsymbol{\mu} \sim \mathrm{SW}^{\boldsymbol{u}}\left(\frac{1}{k}\right)$. Hence

$$
\begin{aligned}
& \underset{\boldsymbol{m}, \boldsymbol{u}, \boldsymbol{\mu}}{\mathbf{E}}\left(\boldsymbol{\mu}_{k}-\alpha_{k} n\right)^{2}=\underset{\boldsymbol{m}, \boldsymbol{u}, \boldsymbol{\mu}}{\mathbf{E}}\left(\boldsymbol{\mu}_{k}-\frac{1}{k} \boldsymbol{u}+\frac{1}{k} \boldsymbol{u}-\alpha_{k} n\right)^{2} \\
\leq & 2 \underset{\boldsymbol{m}, \boldsymbol{u}, \boldsymbol{\mu}}{\mathbf{E}}\left(\boldsymbol{\mu}_{k}-\frac{1}{k} \boldsymbol{u}\right)+2 \underset{\boldsymbol{m}, \boldsymbol{u}}{\mathbf{E}}\left(\frac{1}{k} \boldsymbol{u}-\alpha_{k} n\right)^{2} \leq 2 \underset{\boldsymbol{m}, \boldsymbol{u}, \boldsymbol{\mu}}{\mathbf{E}}\left(\boldsymbol{\mu}_{k}-\frac{1}{k} \boldsymbol{u}\right)^{2}+\frac{2 n \alpha_{k}}{k},
\end{aligned}
$$

where the first inequality used $(x+y)^{2} \leq 2 x^{2}+2 y^{2}$. Given $\boldsymbol{u}$, define $\boldsymbol{v}=\mathrm{E}\left[\boldsymbol{\mu}_{k} \mid \boldsymbol{u}\right]$. Then

$$
\begin{aligned}
& \underset{\boldsymbol{m}, \boldsymbol{u}, \boldsymbol{\mu}}{\mathbf{E}}\left(\boldsymbol{\mu}_{k}-\frac{1}{k} \boldsymbol{u}\right)^{2}=\underset{\boldsymbol{m}, \boldsymbol{u}, \boldsymbol{\mu}}{\mathrm{E}}\left(\boldsymbol{\mu}_{k}-\boldsymbol{v}+\boldsymbol{v}-\frac{1}{k} \boldsymbol{u}\right)^{2} \\
& =\underset{\boldsymbol{m}, \boldsymbol{u}, \boldsymbol{\mu}}{\mathbf{E}}\left[\left(\boldsymbol{\mu}_{k}-\boldsymbol{v}\right)^{2}+\left(\boldsymbol{v}-\frac{1}{k} \boldsymbol{u}\right)^{2}+2\left(\boldsymbol{\mu}_{k}-\boldsymbol{v}\right)\left(\boldsymbol{v}-\frac{1}{k} \boldsymbol{u}\right)\right] \\
& =\underset{\boldsymbol{m}, \boldsymbol{u}, \boldsymbol{\mu}}{\mathbf{E}}\left[\left(\boldsymbol{\mu}_{k}-\boldsymbol{v}\right)^{2}+\left(\boldsymbol{v}-\frac{1}{k} \boldsymbol{u}\right)^{2}\right] \\
& \leq 16 \underset{\boldsymbol{m}, \boldsymbol{u}}{\mathbf{E}} \boldsymbol{u}+\underset{\boldsymbol{m}, \boldsymbol{u}}{\mathbf{E}}\left(\boldsymbol{v}-\frac{1}{k} \boldsymbol{u}\right)^{2}=16 \alpha_{k} k n+\underset{\boldsymbol{m}, \boldsymbol{u}}{\mathbf{E}}\left(\boldsymbol{v}-\frac{1}{k} \boldsymbol{u}\right)^{2},
\end{aligned}
$$

where the inequality uses Proposition 4.8 . Next, we note that because $\boldsymbol{\mu}$ is distributed as $\mathrm{SW}^{\boldsymbol{u}}\left(\frac{1}{k}\right), \boldsymbol{v}$ is at most $\frac{1}{k} \boldsymbol{u}$. Hence, to upperbound $\left(\boldsymbol{v}-\frac{1}{k} \boldsymbol{u}\right)^{2}$ we must lower-bound $\boldsymbol{v}$, and this can be done by Theorem 4.4: $\boldsymbol{v}=\mathrm{E}\left[\boldsymbol{\mu}_{k} \mid \boldsymbol{u}\right] \geq \frac{1}{k} \boldsymbol{u}-2 \sqrt{\boldsymbol{u}}$. Thus, (14) can be bounded as

$$
16 \alpha_{k} k n+\underset{\boldsymbol{m}, \boldsymbol{u}}{\mathbf{E}}\left(\boldsymbol{v}-\frac{1}{k} \boldsymbol{u}\right)^{2} \leq 16 \alpha_{k} k n+\underset{\boldsymbol{m}, \boldsymbol{u}}{\mathbf{E}} 4 \boldsymbol{u}=20 \alpha_{k} k n .
$$

In summary, the term in (11) corresponding to $\overline{\mathcal{G}}$ is at most $42 \alpha_{\geq k} n$.

Using the fact that $\alpha_{k} k, \alpha_{\geq k} \leq \min \left\{1, \alpha_{k} d\right\}$, this implies Theorem 1.5

\subsection{An Alternate Bound on $E_{k}^{(n)}(\alpha)$}

If the gap $\alpha_{k}-\alpha_{k+1}$ is very tiny (or zero), $\operatorname{Excess}_{k}(\alpha)$ will not be a good bound on $E_{k}^{(n)}(\alpha)$. In [OW16] we gave the following bound:

Proposition 4.13. ([OW16, Lemma 5.1].)

$$
\underset{\lambda \sim \mathrm{SW}^{n}(\alpha)}{\mathrm{E}}\left[\underline{\lambda}_{\leq k}-\alpha_{\leq k}\right] \leq \frac{2 \sqrt{2} k}{\sqrt{n}} .
$$

By summing our Theorem 1.4 over all $i \in[k]$, we can replace the constant $2 \sqrt{2}$ by 2 . We now observe that this bound can also be improved so that it tends to 0 with $\alpha_{>k}$.

Proposition 4.14. $\underset{\lambda \sim \mathrm{SW}^{n}(\alpha)}{\mathrm{E}}\left[\underline{\boldsymbol{\lambda}}_{\leq k}-\alpha_{\leq k}\right] \leq O\left(\frac{k \sqrt{\alpha_{>k}}}{\sqrt{n}}\right)$.

Proof. By Theorem 1.14 we may assume that for some $m \geq 1$ we have $\alpha_{k}=\alpha_{k+1}=\alpha_{k+2}=\cdots=\alpha_{k+m-1}$ and $\alpha_{k+m+1}=$ $\alpha_{k+m+2}=\cdots=\alpha_{d}=0$.

Case 1: $m<k$. In this case, Theorem 4.5 tells us that $\mathrm{E}\left[\alpha_{\ell}-\underline{\lambda}_{\ell}\right] \leq$ $2 \sqrt{\alpha_{\ell} \ell / n}$. If $m=1$ then we are done. Otherwise, $\alpha_{k} m \leq 2 \alpha_{>k}$, and so

$$
\mathrm{E}\left[\alpha_{>k}-\underline{\lambda}_{>k}\right] \leq m \cdot 2 \sqrt{2} \sqrt{\alpha_{k} k / n} \leq 2 \sqrt{2} k \sqrt{\alpha_{k} m / n} \leq 4 k \sqrt{\alpha_{>k} / n},
$$

which is equivalent to our desired bound.

Case 2: $m \geq k$. In this case we follow the proof of Proposition 4.13 from [OW16]. Inspecting that proof, we see that in fact the following stronger statement is obtained:

$$
\underset{\lambda \sim \mathrm{SW}^{n}(\alpha)}{\mathrm{E}}\left[\underline{\lambda}_{\leq k}-\alpha_{\leq k}\right] \leq 2 k \sqrt{p_{2} / n}+2 k \sqrt{p_{3} / n},
$$

where it is easy to check (from [OW16, (25)]) that $p_{2}+p_{3}=k \alpha_{k}+$ $\alpha_{>k}$. Now

$$
\sqrt{p_{2}}+\sqrt{p_{3}} \leq 2 \sqrt{p_{2}+p_{3}} \leq 2 \sqrt{m \alpha_{k}+\alpha_{>k}} \leq 2 \sqrt{2 \alpha_{>k}}
$$

where the middle inequality is because we're in Case 2. Combining this with the previous inequality completes the proof.

\section{REFERENCES}

[ANSV08] Koenraad Audenaert, Michael Nussbaum, Arleta Szkoła, and Frank Verstraete. Asymptotic error rates in quantum hypothesis testing. Communications in Mathematical Physics, 279(1):251-283, 2008.

[ARS88] Robert Alicki, Sławomir Rudnicki, and Sławomir Sadowski. Symmetry properties of product states for the system of $N n$-level atoms. Fournal of mathematical physics, 29(5):1158-1162, 1988.

$\left[\mathrm{BAH}^{+} 16\right]$ Michael Beverland, Gorjan Alagic, Jeongwan Haah, Gretchen Campbell, Ana Maria Rey, and Alexey Gorshhkov. Implementing a quantum algorithm for spectrum estimation with alkaline earth atoms. In 19th Conference on Quantum Information Processing, 2016. QIP 2016.

[BDJ99] Jinho Baik, Percy Deift, and Kurt Johansson. On the distribution of the length of the longest increasing subsequence of random permutations. Journal of the American Mathematical Society, 12(4):1119-1178, 1999.

[Bia01] Philippe Biane. Approximate factorization and concentration for characters of symmetric groups. International Mathematics Research Notices, 2001(4):179-192, 2001.

[BL12] Nayantara Bhatnagar and Nathan Linial. On the Lipschitz constant of the RSK correspondence. fournal of Combinatorial Theory, Series A, 119(1):6382, 2012.

[BMW16] Mohammad Bavarian, Saeed Mehraban, and John Wright. Personal communication, 2016.

[BOO00] Alexei Borodin, Andrei Okounkov, and Grigori Olshanski. Asymptotics of plancherel measures for symmetric groups. Fournal of the American Mathematical Society, 13(3):481-515, 2000.

[Buf12] Alexey Bufetov. A central limit theorem for extremal characters of the infinite symmetric group. Functional Analysis and Its Applications, 46(2):8393, 2012. 
[CM06] Matthias Christandl and Graeme Mitchison. The spectra of quantum states and the Kronecker coefficients of the symmetric group. Communications in mathematical physics, 261(3):789-797, 2006.

[FMN13] Valentin Féray, Pierre-Loïc Méliot, and Ashkan Nikeghbali. Mod- $\phi$ convergence I: Normality zones and precise deviations. Technical report, arXiv:1304.2934, 2013.

[Ful97] William Fulton. Young tableaux: with applications to representation theory and geometry. Cambridge University Press, 1997.

[Gre74] Curtis Greene. An extension of Schensted's theorem. Advances in Mathematics, 14:254-265, 1974.

$\left[\mathrm{HHJ}^{+}{ }^{16}\right]$ Jeongwan Haah, Aram Harrow, Zhengfeng Ji, Xiaodi Wu, and Nengkun Yu. Sample-optimal tomography of quantum states. In Proceedings of the 48th Annual ACM Symposium on Theory of Computing, August 2016. Preprint.

[HM02] Masahito Hayashi and Keiji Matsumoto. Quantum universal variablelength source coding. Physical Review A, 66(2):022311, 2002.

$\left[\mathrm{HMR}^{+} 10\right]$ Sean Hallgren, Cristopher Moore, Martin Rötteler, Alexander Russell, and Pranab Sen. Limitations of quantum coset states for graph isomorphism. fournal of the ACM ( (7ACM), 57(6):34, 2010.

[HRTS03] Sean Hallgren, Alexander Russell, and Amnon Ta-Shma. The hidden subgroup problem and quantum computation using group representations SIAM fournal on Computing, 32(4):916-934, 2003.

[HX13] Christian Houdré and Hua Xu. On the limiting shape of Young diagrams associated with inhomogeneous random words. In High Dimensional Probability VI, volume 66 of Progress in Probability, pages 277-302. Springer Basel, 2013.

[IO02] Vladimir Ivanov and Grigori Olshanski. Kerov's central limit theorem for the Plancherel measure on Young diagrams. In Symmetric functions 2001 surveys of developments and perspectives, pages 93-151. Springer, 2002.

[ITW01] Alexander Its, Craig Tracy, and Harold Widom. Random words, Toeplitz determinants and integrable systems I. In Random Matrices and their Applications, pages 245-258. Cambridge University Press, 2001.

[Joh01] Kurt Johansson. Discrete orthogonal polynomial ensembles and the Plancherel measure. Annals of Mathematics, 153(1):259-296, 2001

[Key06] Michael Keyl. Quantum state estimation and large deviations. Reviews in Mathematical Physics, 18(01):19-60, 2006.

[Kup02] Greg Kuperberg. Random words, quantum statistics, central limits, random matrices. Methods and Applications of Analysis, 9(1):99-118, 2002.

[KW01] Michael Keyl and Reinhard Werner. Estimating the spectrum of a density operator. Physical Review A, 64(5):052311, 2001.

[LS77] Benjamin Logan and Larry Shepp. A variational problem for random Young tableaux. Advances in Mathematics, 26(2):206-222, 1977.

[LZ04] Shunlong Luo and Qiang Zhang. Informational distance on quantum-state space. Physical Review A, 69(3):032106, 2004.

[Mél10] Pierre-Loïc Méliot. Kerov's central limit theorem for Schur-Weyl measures of parameter 1/2. Technical report, arXiv:1009.4034, 2010.
[Mél12] Pierre-Loïc Méliot. Fluctuations of central measures on partitions. In 24th International Conference on Formal Power Series and Algebraic Combinatorics, pages 385-396, 2012.

[MM15] Paulina Marian and Tudor Marian. Hellinger distance as a measure of Gaussian discord. Fournal of Physics A: Mathematical and Theoretical, 48(11):115301, 2015.

[MRS08] Cristopher Moore, Alexander Russell, and Leonard Schulman. The symmetric group defies strong Fourier sampling. SIAM fournal on Computing, 37(6):1842-1864, 2008.

[OW15] Ryan O'Donnell and John Wright. Quantum spectrum testing. In Proceedings of the 47th Annual ACM Symposium on Theory of Computing, 2015.

[OW16] Ryan O'Donnell and John Wright. Efficient quantum tomography. In Proceedings of the 48th Annual ACM Symposium on Theory of Computing, 2016.

[Pil90] Shaiy Pilpel. Descending subsequences of random permutations. fournal of Combinatorial Theory, Series A, 53(1):96-116, 1990.

[Rom14] Dan Romik. The surprising mathematics of longest increasing subsequences. Cambridge University Press, 2014.

[Sag01] Bruce E Sagan. The symmetric group: representations, combinatorial algorithms, and symmetric functions. Springer, 2001.

[Sch61] Craige Schensted. Longest increasing and decreasing subsequences. Canadian fournal of Mathematics, 13(2):179-191, 1961.

[TW01] Craig Tracy and Harold Widom. On the distributions of the lengths of the longest monotone subsequences in random words. Probability Theory and Related Fields, 119(3):350-380, 2001.

[Ula61] Stanislaw Ulam. Monte Carlo calculations in problems of mathematical physics. Modern Mathematics for the Engineers, pages 261-281, 1961.

[Vie81] Gérard Viennot. Équidistribution des permutations ayant une forme donnée selon les avances et coavances. Fournal of Combinatorial Theory. Series A, 31(1):43-55, 1981.

[VK77] Anatoly Vershik and Sergei Kerov. Asymptotic behavior of the Plancherel measure of the symmetric group and the limit form of Young tableaux. Soviet Mathematics Doklady, 18:118-121, 1977.

[VK81] Anatoly Vershik and Sergei Kerov. Asymptotic theory of characters of the symmetric group. Functional analysis and its applications, 15(4):246-255, 1981.

[VK85] Anatoly Vershik and Sergei Kerov. Asymptotic of the largest and the typical dimensions of irreducible representations of a symmetric group. Functional Analysis and its Applications, 19(1):21-31, 1985.

[Wer94] Lorenz Wernisch. Dominance relation on point sets and aligned rectangles. $\mathrm{PhD}$ thesis, Free University Berlin, 1994.

[WY16] Yihong Wu and Pengkun Yang. Minimax rates of entropy estimation on large alphabets via best polynomial approximation. IEEE Transactions on Information Theory, 62(6):3702-3720, 2016. 